\title{
Dynamic Effect in the Capillary Pressure-Saturation Relationship and its Impacts on Unsaturated Flow
}

\author{
S. Majid Hassanizadeh,* Michael A. Celia, and Helge K. Dahle
}

\begin{abstract}
Capillary pressure plays a central role in the description of water flow in unsaturated soils. While capillarity is ubiquitous in unsaturated analyses, the theoretical basis and practical implications of capillarity in soils remain poorly understood. In most traditional treatments of capillary pressure, it is defined as the difference between pressures of phases, in this case air and water, and is assumed to be a function of saturation. Recent theories have indicated that capillary pressure should be given a more general thermodynamic definition, and its functional dependence should be generalized to include dynamic effects. Experimental evidence has slowly accumulated in the past decades to support a more general description of capillary pressure that includes dynamic effects. A review of these experiments shows that the coefficient arising in the theoretical analysis can be estimated from the reported data. The calculated values range from $10^{4}$ to $10^{7} \mathrm{~kg}$ $(\mathrm{m} \mathrm{s})^{-1}$. In addition, recently developed pore-scale models that simulate interface dynamics within a network of pores can also be used to estimate the appropriate dynamic coefficients. Analyses of experiments reported in the literature, and of simulations based on porescale models, indicate a range of dynamic coefficients that spans about three orders of magnitude. To examine whether these coefficients have any practical effects on larger-scale problems, continuum-scale simulators may be constructed in which the dynamic effects are included. These simulators may then be run to determine the range of coefficients for which discernable effects occur. Results from such simulations indicate that measured values of dynamic coefficients are within one order of magnitude of those values that produce significant effects in field simulations. This indicates that dynamic effects may be important for some field situations, and numerical simulators for unsaturated flow should generally include the additional term(s) associated with dynamic capillary pressure.
\end{abstract}

$\mathrm{C}$ APILLARITY plays a central role in the description of multiphase (and unsaturated) flow in porous media. In quantitative modeling of multiphase flow, a relationship is needed to describe capillary pressure as a function of other medium properties. Although the underlying processes that determine the distribution of fluid phases in porous media are extremely complicated, the main theoretical and practical tool currently used to quantify the capillary pressure function is an empirical relationship between capillary pressure and saturation in the form (see, e.g., Bear and Verruijt, 1987):

$$
P^{\mathrm{n}}-P^{\mathrm{w}}=P^{\mathrm{c}}=f(S)
$$

S.M. Hassanizadeh, Section for Hydrology and Ecology; Faculty of Civil Engineering and Geosciences, Delft University of Technology; P.O. Box 5048, 2600GA Delft, The Netherlands; Michael A. Celia, Environmental Engineering and Water Resources Program, Department of Civil and Environmental Engineering, Princeton University; Princeton, NJ 08544, USA; Helge K. Dahle, Department of Mathematics, University of Bergen, Johannes Brunsgate 12, 5008 Bergen, Norway. Received 26 Nov. 2001. *Corresponding author (majid@citg. tudelft.nl)

Published in Vadose Zone Journal 1:38-57 (2002). where $P^{\mathrm{n}}$ and $P^{\mathrm{w}}$ are the average pressures of nonwetting and wetting phases, respectively; $P^{\mathrm{c}}$ is capillary pressure, and $S$ is the wetting phase saturation. A schematic depiction of $P^{\mathrm{c}}$ vs. $S$ curves is given in Fig. 1.

This simple model is implicitly assumed to account for all effects and processes that influence the equilibrium distribution of fluids, such as surface tension, presence of fluid-fluid interfaces, wettability of solid surfaces, grain size distribution, and microscale heterogeneities. All of these effects are essentially lumped into the $P^{\mathrm{c}}-S$ relationship. Moreover, this relationship and graphs similar to those depicted in Fig. 1 are obtained experimentally under equilibrium conditions. Thus, to obtain a drainage (or imbibition) curve, one starts with a wet (or dry) soil sample, then the capillary pressure is increased (or decreased) incrementally, and at each step the water content is measured after equilibrium is reached. The time to equilibrium after each imposed pressure increment ranges from a few hours to many days, depending on the soil type and saturation degree (see, e.g., Elrick, 1963; Stephens, 1995, p. 189). The typical time needed to construct a complete capillary pressure-saturation curve is weeks or longer. Now, the question arises whether such curves adequately describe the relationship between $P^{\mathrm{n}}-P^{\mathrm{w}}$ and $S$ in drainage or imbibition events with a time scale in the order of hours. In fact, there is ample theoretical and experimental evidence that this simple relationship is not unique, but it depends on the flow dynamics - it depends on both the history and the rate of change of saturation. The dependence of capillary pressure-saturation curves on the history of flow is known as capillary pressure hysteresis; this is a well-known effect and has been the subject of extensive investigations. The dependence of capillary curves on the rate of change of saturation is due to dynamic effects. It is much less known and is not quantified properly. The latter effect is the subject of this study.

Another important parameter in the description of unsaturated flow is relative permeability, which is also considered to be a function of saturation. There are some indications that the relative permeability-saturation relationship also shows hysteresis effects and may depend on the rate of change of saturation. These effects, however, are less pronounced than in the case of capillary pressure. It must be noted that the dynamic effect considered in this paper is different from the flow-rate dependence of the relative permeability coefficient. It is known that relative permeability shows some dependence on capillary number (basically on the rate of fluid flow). This dependence is more significant at high capillary numbers and is present under steady-state conditions as well (see, e.g., Blom and Hagoort, 1997; Ramakrishnan and Wasan, 1986). However, the dynamic effect, related to the rate of change of saturation, is a transient 
effect that may be significant even for small capillary numbers, and is not present under steady-state flow, as discussed later. Also we note that we are not addressing the validity of Darcy's Law that specifies a linear relationship between flow rate and pressure gradient. In this paper, we assume that Darcy's Law holds.

In this work, we first present a dynamic capillary pressure-saturation relationship that has been obtained in the framework of a macroscopic theory of porous media flow developed by Hassanizadeh and Gray (1990). Next, we give an overview of experiments reported in the literature that include evidence of dynamic effects. A review of some of the early works in this area has been given by Wanna-Etyem (1982). Some of those works are mentioned here too for the sake of completeness and also because we analyze them differently. We argue that abnormalities in soil characteristic curves observed in various experiments can be explained by the new theory of dynamic capillary pressure presented here. The magnitude of the new dynamic capillary pressure coefficient is determined from available data. Next, a dynamic pore-scale network model is employed to investigate the link between macroscale dynamic effects and pore-scale flow processes. Finally, the potential significance of the dynamic effect on the redistribution of moisture in unsaturated soil is investigated by means of a numerical model. It is shown that the dynamic effect can lead to significant retardation of infiltration fronts as compared with the standard static capillary pressure models.

\section{DYNAMIC CAPILLARY PRESSURE-SATURATION RELATIONSHIP}

The standard relationship assumed between capillary pressure and saturation is empirical in nature, and as such, it lacks a firm theoretical foundation. To overcome this deficiency, the definition of macroscopic capillary pressure must be developed in the framework of a sound thermodynamic theory. To achieve this, one must provide macroscopic balance laws and appropriate constitutive relationships for interfacial as well as phase properties of the porous medium. Such an approach has been developed and successfully applied to the modeling of two-phase flow (Hassanizadeh and Gray, 1990, 1993a,b; Gray and Hassanizadeh, 1991a,b). As a result, a thermodynamic theory of two-phase flow in a porous medium has been produced which has the aforementioned characteristics. The main constitutive hypothesis in this theory is the dependence of the Helmholtz free energy functions for the phases and interfaces on state variables such as mass density, temperature, saturation, porosity, interfacial area density, and the solid phase strain tensor. Explicit inclusion of interfaces and interfacial properties in the macroscale theory of two-phase flow is an essential characteristic of the new approach. Interfaces are known to have a significant role in determining the thermodynamic state of the whole system (see, e.g., Kirkham

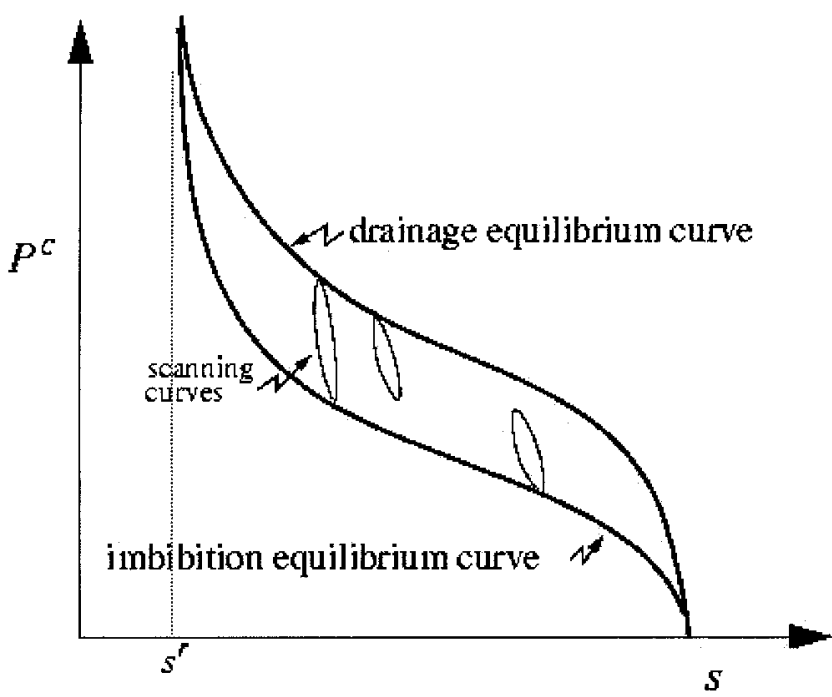

Fig. 1. Typical quasistatic plot of capillary pressure vs. saturation.

and Feng, 1949; Dussan, 1987). In the case of two-phase flow in a porous medium, three phases and three interfaces are involved. These are typically a wetting phase, a nonwetting phase, and the solid phase, here denoted by superscripts $\mathrm{w}, \mathrm{n}$, and $\mathrm{s}$. In addition, a typical interface is denoted by $\alpha \beta$, which may take on values wn, ws, and ns. The following results of the constitutive theory for fluid pressures and for capillary pressure (Hassanizadeh and Gray, 1993a) are of interest here:

$$
\begin{gathered}
P^{\alpha}=\left(\rho^{\alpha}\right)^{2} \frac{\partial A^{\alpha}}{\partial \rho^{\alpha}} \quad \alpha=w, n \\
P^{\mathrm{c}}=-S \rho^{\mathrm{w}} \frac{\partial A^{\mathrm{w}}}{\partial S}+(1-S) \rho^{\mathrm{n}} \frac{\partial A^{\mathrm{n}}}{\partial S}-\sum_{\alpha \beta} \frac{\gamma^{\alpha \beta}}{\varepsilon}\left(\frac{\partial a^{\alpha \beta}}{\partial S}\right)
\end{gathered}
$$

where $A^{\alpha}$ is Helmholtz free energy of phase per unit mass of phase, $\rho^{\alpha}$ is mass of $\alpha$ phase per unit volume of $\alpha$ phase, $\varepsilon$ is porosity, $S$ is saturation of the wetting phase, $a^{\alpha \beta}$ is area of $\alpha \beta$ interface per unit volume of porous medium, $\gamma^{\alpha \beta}$ is interfacial tension of the $\alpha \beta$ interface, and $\Sigma_{\alpha \beta}$ denotes a summation over all interfaces.

The definition (Eq. [2]) for fluid pressures is similar to the one given in classical thermodynamics of single phases. The definition (Eq. [3]) for capillary pressure, however, is new. Here, capillary pressure is defined to be equal to the change in the free energy of the system as a result of a change in the wetting phase saturation. Accordingly, capillary pressure provides an indication of the tendency of the system to admit a change in the saturation of the wetting phase when temperature, density, and porosity are held constant.

Equation [3] explains the spontaneity of an imbibition process. According to this equation, and given the restriction that capillary pressure has to be positive, the free energy of the system must decrease for the saturation of the wetting phase to increase (i.e., for $\Delta S>0$ ); this would be a spontaneous process. On the other hand, to decrease the wetting phase saturation $(\Delta S<0)$, the energy of the system must be increased. This will not be a spontaneous process, and it will be possible only if external work is performed. In this approach, macro- 
scopic capillary pressure is thus defined solely as an intrinsic property of the system and is not simply equal to the difference in fluid phases pressures. Note that $P^{\mathrm{c}}$ is only a measure of the tendency of the system to undergo a change in saturation. Whether this change will actually occur depends on initial and boundary conditions, on the overall state of the system, and in particular, on the pressure distribution of the two fluids. This is mathematically stated by the residual entropy inequality. It can be shown (Hassanizadeh and Gray, 1993a) that the following combination of terms contributes to the entropy production such that, in the absence of other thermodynamic forces, one must have:

$$
-\varepsilon \frac{D^{\mathrm{s} S}}{D t}\left[\left(P^{\mathrm{n}}-P^{\mathrm{w}}\right)-\left(P^{\mathrm{c}}\right)\right] \geq 0
$$

where $\mathrm{D}^{\mathrm{s}} \mathrm{S} / \mathrm{D} t$ is the material time derivative of the wetting phase saturation observed when moving with the solid phase. For a nondeforming porous medium, this derivative reduces to the partial time derivative of saturation $\partial S / \partial \mathrm{t}$.

In inequality (Eq. [4]), $P^{\mathrm{n}}-P^{\mathrm{w}}$ is the resultant of external forces causing the movement of fluids in the porous medium. This movement is opposed (or assisted) by capillary forces. Under equilibrium or steady-state conditions (i.e., $\mathrm{D}^{s} S / \mathrm{D} t=0$ ), the capillary forces will balance $P^{\mathrm{n}}-P^{\mathrm{w}}$ so that one will have:

$$
P^{\mathrm{n}}-P^{\mathrm{w}}=P^{\mathrm{c}}(S)
$$

Inequality (Eq. [4]) indicates that if equilibrium is disturbed such that $P^{\mathrm{n}}-P^{\mathrm{w}}>P^{\mathrm{c}}$ (e.g., because of a decrease in $P^{\mathrm{w}}$ and/or an increase in $P^{\mathrm{n}}$ ), then $\mathrm{D}^{s} S / \mathrm{D} t$ must be negative; this corresponds to drainage taking place. On the other hand, if $P^{\mathrm{n}}-P^{\mathrm{w}}<P^{\mathrm{c}}$, then $\mathrm{D}^{s} S / \mathrm{D} t$ must be positive; this corresponds to imbibition taking place. Only at equilibrium, when $\mathrm{D}^{s} S / \mathrm{D} t=0$ and no change in saturation is occurring, will $P^{\mathrm{n}}-P^{\mathrm{w}}=P^{\mathrm{c}}$. Obviously, the rate at which drainage or imbibition takes place depends on the magnitude of the difference $\left(P^{\mathrm{n}}-P^{\mathrm{w}}\right)-P^{\mathrm{c}}$. For a linear theory, the following approximation for $\mathrm{D}^{s} \mathrm{~S} / \mathrm{D} t$ may be given (Hassanizadeh and Gray, 1993a)

$$
\left.-\varepsilon \frac{\mathrm{D}^{\mathrm{s}} S}{\mathrm{D} t}=-(\tau)^{-1}\left(P^{\mathrm{n}}-P^{\mathrm{w}}\right)-P^{\mathrm{c}}(S)\right]
$$

where $\tau\left(\mathrm{M} \mathrm{L}^{-1} \mathrm{~T}^{-1}\right)$ is a material coefficient that may still depend on saturation. Substitution of Eq. [6] in Eq. [4] will reveal that $\tau$ must not be negative. Both the general inequality (Eq. [4]) and the approximation (Eq. [6]) suggest that at a given point in the system and at any given time, saturation will change locally in order to restore equilibrium; that is, the equivalence between $P^{\mathrm{n}}-P^{\mathrm{w}}$ and $P^{\mathrm{c}}$.

Equation [6] suggests that if we were to plot $P^{\mathrm{n}}-P^{\mathrm{w}}$ as a function of saturation, the resulting curve would also depend on $\mathrm{D}^{s} S / \mathrm{D} t$. The overview of experiments in the next section shows that the traditional capillary pressure-saturation curves are indeed a function of $\mathrm{D}^{s} S /$ $\mathrm{D} t$. The coefficient $\tau$ may be interpreted as a measure of the speed with which a change in saturation takes place. If $\tau$ is found to be small, equivalence between $P^{\mathrm{n}}-$ $P^{\mathrm{w}}$ and $P^{\mathrm{c}}$ will be reestablished virtually instantaneously after equilibrium is disturbed. Therefore, $\tau$ behaves as a capillary damping coefficient. If one employs the traditional relation (Eq. [5]) for the definition of macroscopic capillary pressure, one is implicitly assuming that any disturbances at equilibrium are eliminated almost instantaneously. System dynamics such as these are probably achieved only for porous media with good permeability. As permeability decreases, the applicability of Eq. [5] at nonequilibrium will become suspect. In cases where permeability of the medium to the wetting phase is low, Eq. [5] will apply only at equilibrium or steady state, and one probably needs to employ an equation such as Eq. [6] to correctly model system dynamics.

It must be noted that $P^{\mathrm{c}}(S)$ in Eq. [5] and [6] may still be a hysteretic function of saturation. In other words, the dynamic term in Eq. [6] (or its other forms presented below) does not account for hysteresis. Even the coefficient $\tau$ may have a hysteretic character; having different values at drainage and imbibition. This needs to be established experimentally. This theory has been recently extended to include hysteretic effects in both $P^{\complement}(S)$ and $\tau$ by Beliaev and Hassanizadeh (2001). The extended theory has been investigated numerically by Beliaev and Schotting (2002).

For the purpose of studying experiments reported in the literature, Eq. [6] is cast into a slightly different form. The pressure difference $P^{\mathrm{n}}-P^{\mathrm{w}}$, which is the commonly measured quantity, is denoted as $P_{\mathrm{dyn}}^{\mathrm{c}}$ and the equilibrium (or "static") capillary pressure $P^{\mathrm{c}}$ is denoted as $P_{\text {stat }}^{\mathrm{c}}$ so that Eq. [6] becomes:

$$
P_{\mathrm{dyn}}^{\mathrm{c}}-P_{\text {stat }}^{\mathrm{c}}=-\tau \frac{\partial S}{\partial t}
$$

where the material time derivative is approximated by the partial time derivative. For unsaturated flow, where the air pressure is equal to the constant atmospheric pressure, this equation may be written in terms of the water pressure head $\Psi=P^{\mathrm{w}} / \rho g$ :

$$
\Psi_{\text {dyn }}-\Psi_{\text {stat }}=\frac{\tau}{\varepsilon \rho g}\left(\frac{\partial \theta}{\partial t}\right)
$$

where $\theta=\varepsilon S$ is volumetric water content, $\rho$ is water mass density, and $g$ is the gravity. In this work, variations in water mass density and soil porosity are not considered, and therefore, the terms capillary pressure and capillary head (or water gauge pressure head in the case of unsaturated flow), and water saturation and water content, are used interchangeably. Thus, we may refer to soil characteristic curves as capillary pressure-saturation or capillary head-water content curves.

\section{OVERVIEW OF EXPERIMENTS}

Equation [7] suggests that dynamic capillary pressure is larger than static capillary pressure in drainage and smaller in wetting. This supposition has been found true, in full or partially, by a number of experimental works during the past 50 years. Most of the experiments reported here were not designed to look for dynamic 
effects, but instead were aimed at testing the validity of the Richards equation, identifying factors that affect soil moisture characteristics, or addressing parameter estimation issues.

The first indications of nonuniqueness of soil moisture characteristic curves (probably due to dynamic effects) seem to have been reported by Kirkham and Feng (1949). Many experiments followed which, we believe, contain evidence of the presence of dynamic effects. Most researchers suggested factors other than dynamic effect as the reasons for the observed deviations.

In this section, a brief description of some of the experimental works and results obtained is given. In each case, only those experiments directly relating to dynamic effects are described; experiments relating to effects of, for example, temperature or solute concentration, are not discussed. Almost all experiments we have found in the literature relate to unsaturated flow. The only exception is the work of Kalaydjian (1992b), who performed imbibition experiments, where water displaced oil, and found very pronounced dynamic effects.

Unsaturated flow of water in a horizontal soil column is given by a diffusion type equation:

$$
\frac{\partial \theta}{\partial \mathrm{t}}=\frac{\partial}{\partial x}\left[k_{\mathrm{r}}(\theta) K \frac{\partial \Psi_{\mathrm{dyn}}}{\partial x}\right]
$$

where $K$ is saturated hydraulic conductivity and $k_{\mathrm{r}}$ is the relative permeability coefficient, assumed to be a function of water content. The subscript "dyn" has been added to stress the fact that the pressure head measured under flow conditions is used in Darcy's Law. Commonly, the standard capillary pressure-saturation relationship, measured under equilibrium conditions, is used to rewrite Eq. [9] in terms of water content. That is, one assumes:

$$
\Psi_{\text {dyn }}=\Psi_{\text {stat }}=\Psi(\theta)
$$

As a result, Eq. [9] may be written in the form of a diffusion equation:

$$
\frac{\partial \theta}{\partial \mathrm{t}}=\frac{\partial}{\partial x}\left[D(\theta) \frac{\partial \theta}{\partial x}\right]
$$

where $D$ denotes moisture diffusivity, defined by:

$$
D=k_{\mathrm{r}}(\theta) K \frac{\partial \Psi}{\partial \theta}
$$

If one is to differentiate between $\Psi_{\text {dyn }}$ or $\Psi_{\text {stat }}$, then, depending on which one is used in Eq. [12], one will obtain different diffusivities that may be denoted with $D_{\text {dyn }}$ or $D_{\text {stat }}$.

\section{Experiments on the Validity of the Moisture Diffusion Equation}

Early experiments focused on testing the validity of diffusion Eq. [11] (see Kirkham and Feng, 1949; Biggar and Taylor, 1960; Nielsen et al., 1962; Rawlins and Gardner, 1963). In those experiments, the advance of a wetting front into a horizontal column filled with dry soil was monitored and the amount of water flux into the column as a function of time was measured. Diffusion
Eq. [11] prescribes that the distance $x$ traveled by the wetting front, as well as the flux of water entering the column, $Q$, should be a linear function of $\sqrt{t}$ (see Crank, 1956, p. 36):

$$
Q \propto \sqrt{t} \quad \text { and } \quad x \propto \sqrt{t}
$$

Kirkham and Feng (1949) carried out wetting experiments on soil samples of clay loam, silt loam, and sand, with permeabilities varying from low to high. In all experiments, the sample was initially air dried with a saturation of about $5 \%$. Water was allowed to enter the soil sample at a suction of about $1 \mathrm{~cm}$. The volume of water entering the sample, $Q$, was measured as a function of time. The distance of the wet-dry front from the entrance, $x$, was visually determined and plotted as a function of time. They indeed found that both $Q$ and $x$ vary linearly with the square root of time. However, the lines did not go through the origin (similar to lines in Fig. 2). Also, when curves of measured moisture content vs. distance were compared with curves calculated with the aid of Eq. [11], significant disagreement was found. Kirkham and Feng (1949) concluded that the diffusion equation is deficient in describing unsaturated flow.

Similar wetting experiments were carried out by Biggar and Taylor (1960) on silt loam samples and similar results were obtained. Moreover, in different experiments, they imposed different suction values on the water entering dry soil, ranging from 25 to $100 \mathrm{~cm}$. Plots of the amount of water entering the soil as a function of square root of time, at different values of imposed suction are given in Fig. 2. As mentioned above, the slope of these lines is directly related to the diffusivity coefficient. Thus, Biggar and Taylor (1960) determined that, for a given soil, the diffusivity increases as the source water suction decreases. A low value of the source suction corresponds to a larger moisture content at the boundary, and thus a larger driving force and, therefore, a faster infiltration (or a larger value of $\partial \theta / \partial t$ ). Note that this result is different from the fact that diffusivity depends on the soil water content (or saturation). This experiment demonstrates that, contrary to the common understanding, diffusivity is not an intrinsic property of the soil, but it depends on how fast the wetting process occurs.

Similar results were obtained in a set of extensive and careful experiments by Nielsen et al. (1962). They too carried out wetting experiments on air-dried samples of clay loam, silt loam, and sandy loam, with the water applied at a range of suction values $(2,50$, or $100 \mathrm{~cm})$. Moreover, they used oil as the wetting fluid in some of the experiments with soil and did wetting experiments with sandstone instead of soil. Experiments involving oil or sandstone proved that the observed results were not caused by soil swelling or shrinking, or any other structural instability. Nielsen et al. (1962) calculated diffusivity as function of water content from experiments with the same soil wetted at different water suctions (see Fig. 3). Here again they found a larger diffusivity at higher wetting rates. Nielsen et al. concluded that the diffusion equation could not be verified for moisture transport. This implies, they argued, that either Darcy's 


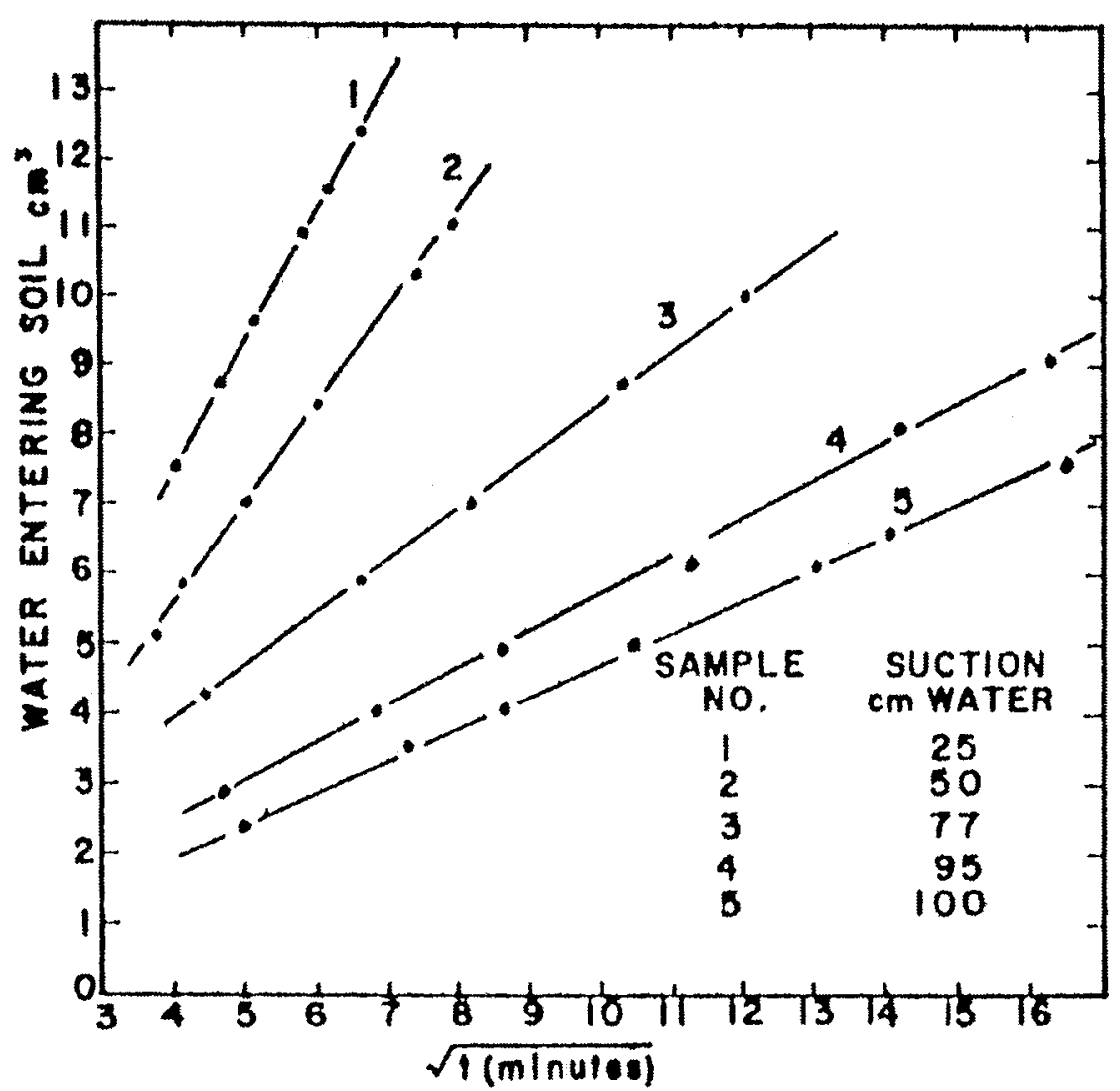

Fig. 2. Total amount of water entering dry soil as a function of square root of time, at different values of imposed suction; from Biggar and Taylor (1960)

Law does not apply to unsaturated flow or diffusivity is not a unique function of moisture content.

Similar conclusions were reached by Rawlins and Gardner (1963) in yet another set of wetting experiments with samples of silty clay loam. They evacuated the soil to eliminate air entrapment and to avoid air pressure buildup ahead of the wetting front. They also found that, assuming Darcy's Law to be valid, diffusivity is not a unique function of water content. Their plot of diffusivity vs. water content calculated at different times during the wetting process is given in Fig. 4. Rawlins and Gardner were not sure of the causes of this nonuniqueness. They pointed out that it cannot be due to hysteresis because the soil remained at all times under wetting conditions in their experiment. Also, they believed variations in bulk density, pore geometry (e.g., due to swelling), and temperature, although present, were not significantly large to cause the kind of systematic deviations from uniqueness observed in their experiments.

Regardless of the mechanisms underlying these dynamic effects, the nonuniqueness of the diffusivity can be explained by a rate dependence of either $k_{\mathrm{r}}(\theta)$ or $\Psi(\theta)$ or both. If, for the sake of argument, we assume that dynamic effects on $k_{\mathrm{r}}(\theta)$ are negligible, then the sense of nonuniqueness observed in the above-mentioned works is in line with the sense of Eq. [8]. According to this equation, under wetting conditions, $\Psi_{\text {dyn }}$ should be bigger than $\Psi_{\text {stat }}$. Results given in Fig. 4 show this to be the case. This can be made evident by noting that $\Psi$ is directly related to the area under the diffusivity curve $D(\theta)$, a result that follows from integration of Eq. [12]. The area under the diffusivity curve estimated at early times (which gives $\Psi_{\text {dyn }}$ ), is larger than the area under the diffusivity curve estimated at later times when $\partial \theta / \partial t$ is small (which gives $\Psi_{\text {stat }}$ ).

\section{Experiments on the Uniqueness of $\Psi-\theta$ or $\boldsymbol{P}^{\mathbf{c}}-\boldsymbol{S}$ Relationship}

One of the questions that remained unanswered in the experiments described above was whether the nonuniqueness in diffusivity was due to a nonuniqueness in $\Psi(\theta)$ and/or in $k_{\mathrm{r}}(\theta)$. The experiments discussed below were designed to investigate this question. Most experiments are focused on the uniqueness of the $\Psi-\theta$ relationship. Some also looked into the presence of dynamic effects in the $k_{\mathrm{r}}-\theta$ relationship (see Elzeftawy and Mansell, 1975). As explained in the Introduction, capillary pressure curves, similar to those in Fig. 1, are obtained as best fit to equilibrium points. In the experiments discussed below, large pressure changes were imposed on the soil samples and then the water pressure head and water content were measured under dynamic (flow) conditions.

Perhaps the first to look into the nonuniqueness of the $\Psi-\theta$ relationship were Mokady and Low (1964). On the basis of thermodynamic arguments, they argued that the $\Psi-\theta$ relationship cannot be unique. They hypothesized that, at a given water content under drainage con- 


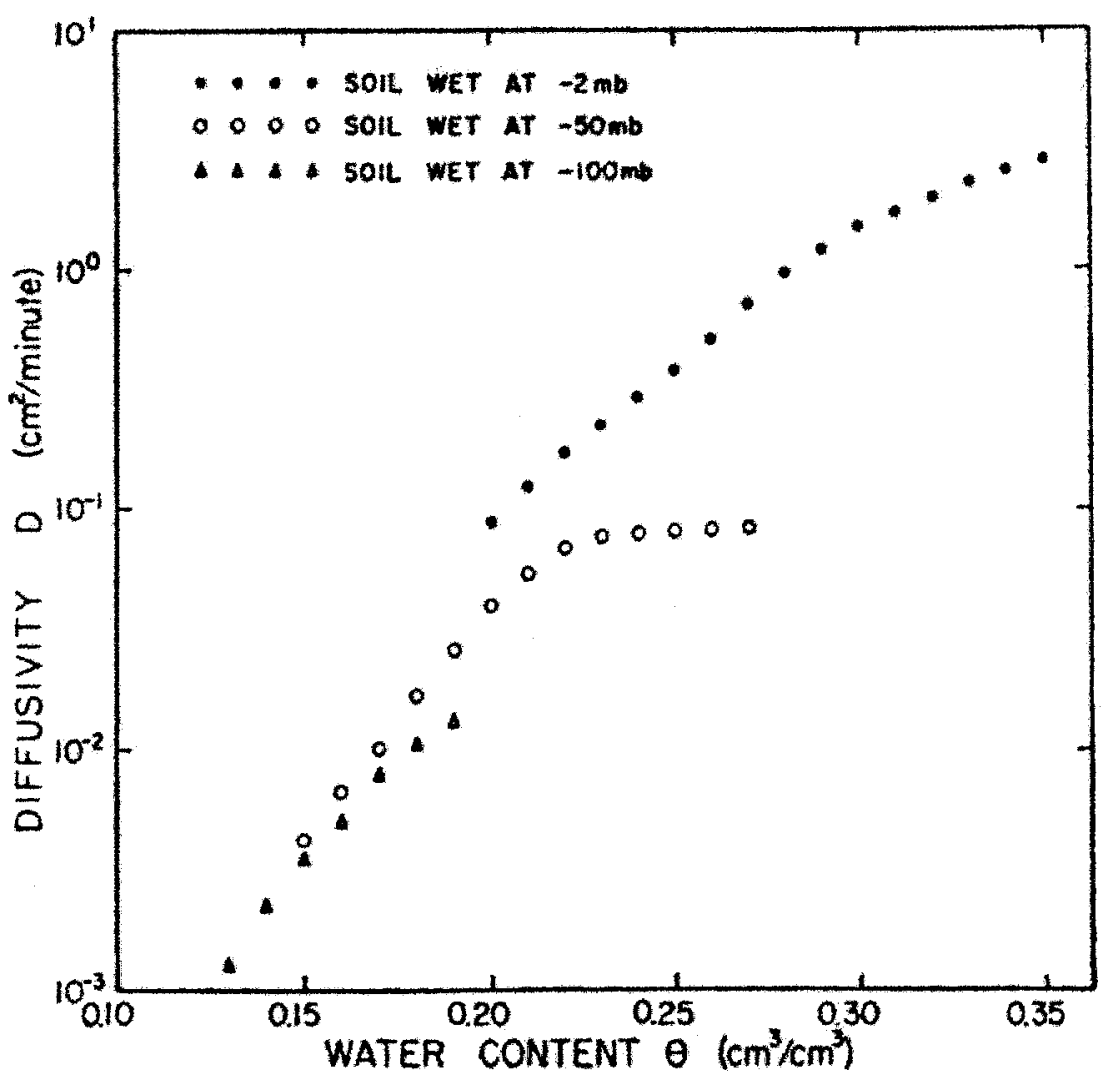

Fig. 3. Diffusivity as function of water content estimated by Nielsen et al. (1962) from experiments wherein dry soil was wetted at different water suctions.

ditions, the transient capillary head will be smaller than the equilibrium head. This, however, was later proven to be incorrect; the effect is the other way around. Mokady and Low (1964) carried out experiments on a soil sample composed of a mixture of $75 \%$ banding sand, $12.5 \%$ silica flour, and $12.5 \%$ kaolinite. They carried both dynamic and drainage experiments in which the saturation was reduced from 100 to $97 \%$. They did not find any significant difference between static and dynamic data. They argued that the absence of dynamic effects was mainly because of the very small range of saturation change. They contended that for larger changes in saturation, one would find different $\Psi-\theta$ curves under dynamic and static conditions. Later on, Watson (1965) carried out drainage experiments on coarse sand and reported that the $\Psi-\theta$ relationship was independent of the state of flow. But, shortly thereafter, experiments by Davidson et al. (1966) provided the first indications of differences between the $\Psi-\theta$ relationships under static and dynamic conditions. This was soon confirmed by Watson and Whisler (1968) and others, as described below.

Topp et al. (1967) carried out drainage experiments on a sandy soil with size fractions ranging from 0.10 to $0.5 \mathrm{~mm}$. Dry soil was packed into a rectangular container 2.54 by 1.0 by $7.6 \mathrm{~cm}$ high. The soil had a bulk density of $1.69 \mathrm{~g} \mathrm{~cm}^{-3}$ and a total porosity of 0.365 . No attempt was made to check the uniformity of packing from point to point. Water content and pressure head were measured at a point in the middle of the column simultaneously. Water content was measured by means of a gamma ray attenuation apparatus. Capillary pressure head and hydraulic gradient were measured by means of tensiometer plates. The sample was first flushed with $\mathrm{CO}_{2}$ and then saturated with an air-free water solution of $0.005 \mathrm{~N} \mathrm{CaSO}_{4}$ and $0.005 \mathrm{~N} \mathrm{NaCl}$. The same water solution (with a total volume of about 100 times the

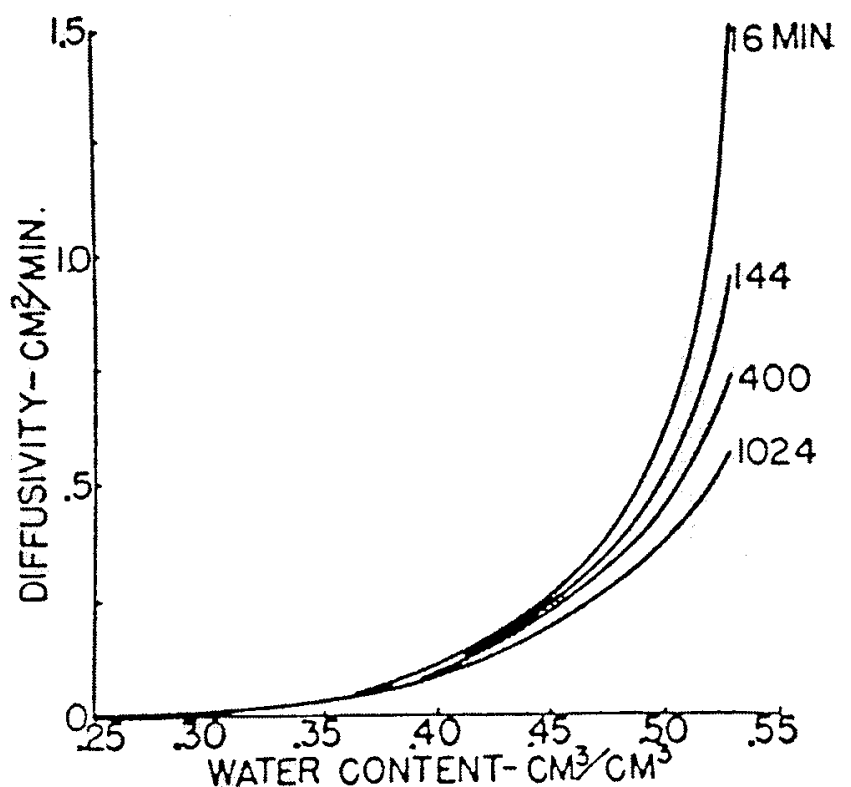

Fig. 4. Diffusivity as function of water content estimated by Rawlins and Gardner (1963) from experiments wherein dry soil was wetted at different water suctions. 


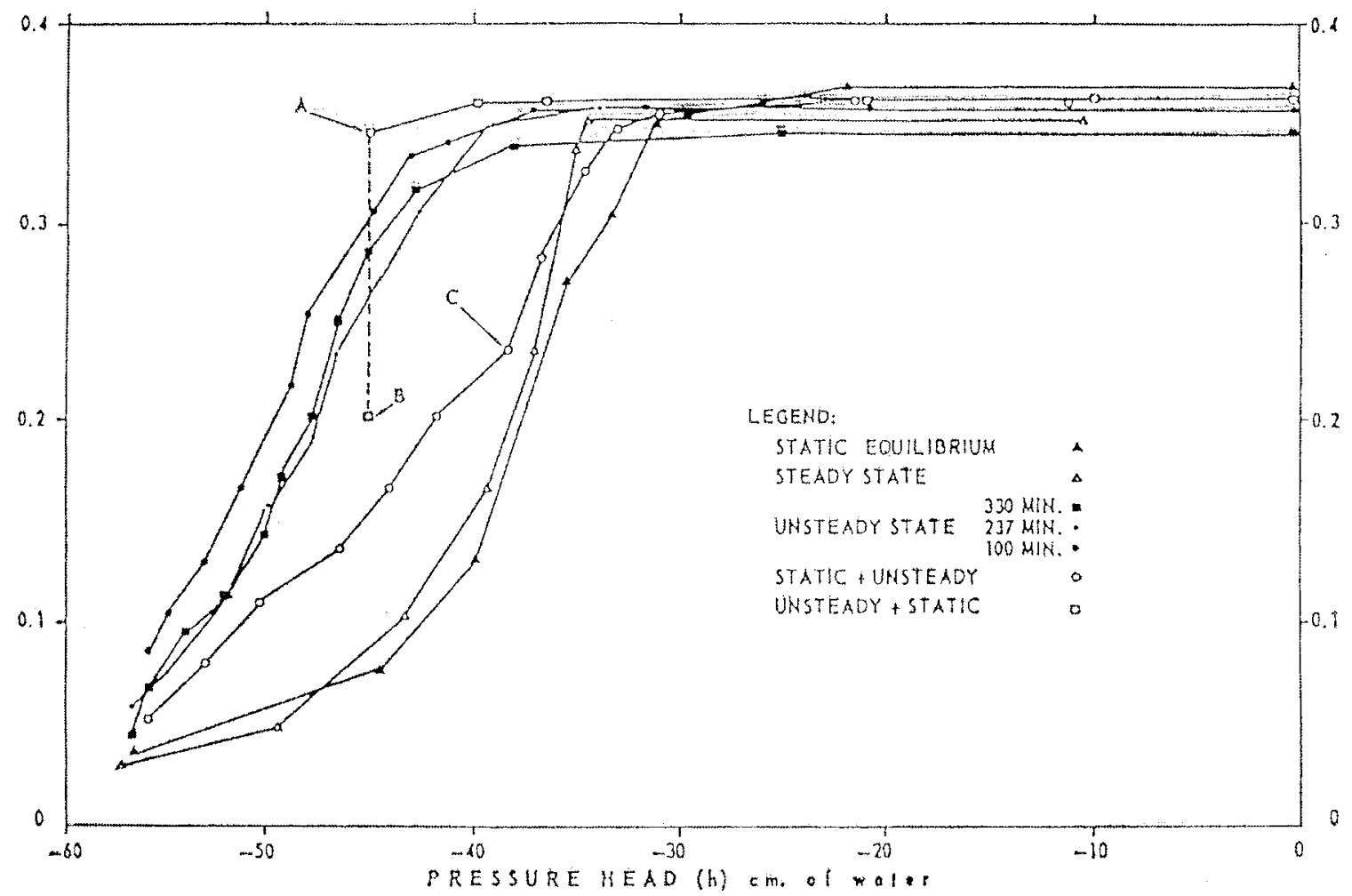

Fig. 5. Water content-pressure head data from drainage experiments of Topp et al. (1967).

sample volume) was used throughout all experiments. A gas inlet slit was located along the top end of the sample. The water content was varied by controlling the gas phase pressure and allowing water to leave or enter the sample from the bottom of the column. The drainage water content-capillary head relationship, starting at saturation, was determined by one of the following methods: (i) static equilibrium, (ii) steadystate flow, or (iii) unsteady-state flow. In each method, the sample was drained until a capillary pressure head of about $56 \mathrm{~cm}$ was reached. The sample was then saturated again by introducing and maintaining a flow of air-free solution. Plots of water content as a function of capillary pressure head are given in Fig. 5. In the static equilibrium procedure, a series of equilibrium states was established at successively higher gas phase pressures. The time required to reach equilibrium at a given water content was around 1000 and $4000 \mathrm{~min}$ for high and low water contents, respectively. In the steady-state method, vertical downward steady-state flow was established in the sample at approximately unit hydraulic gradient at a series of increasing gas phase pressures. The time required to reach steady state at a given water content varied between 300 and 600 min. As seen in Fig. 5, data points from equilibrium and steady-state experiments fall very close to each other. Based on our theory, this result should be expected because in both cases the rate of change of saturation is negligibly small $(\partial \theta / \partial t \approx 0)$ and thus dynamic effects are not present. In the unsteady-state experiments, starting from the saturated state, an outflow of water from the sample was induced by increasing the gas pressure in steps of 1 to $2 \mathrm{~cm}$ of water at frequent time intervals. The total time used to go from the saturated state to a capillary pressure of $56 \mathrm{~cm}$ of water was varied in three different runs from 100 to 237 to $330 \mathrm{~min}$. These obviously correspond to significantly large values of $\partial \theta / \partial t$. As it can be seen from the plot of Fig. 5 for the unsteady-state runs, the (dynamic) capillary pressure is significantly higher than in the case of equilibrium or steady-state runs. This is clearly in line with the prediction of Eq. [10] for drainage. Topp et al. (1967) carried out additional experiments in which the flow was vertically upward (instead of downward) or the air pressure was kept constant and the water pressure was reduced. In all cases, results similar to those shown here were obtained. Topp et al. (1967) were not sure about the sources of this nonuniqueness in capillary pressure-water content curves. As possible causes, they mention the presence of disconnected pendular water rings or the accumulation of traces of contaminants in the air-water interfaces within the sample. However, they do not make any attempt to quantify the significance of these effects.

In a series of experiments by Vachaud, Vauclin, and coworkers, similar dynamic effects were observed. Smiles et al. (1971) carried out both wetting and drainage experiments on a sandy soil, where the moisture movement took place horizontally. Dry sand was packed into a plexiglass tube $60 \mathrm{~cm}$ long and $5 \mathrm{~cm}$ in diameter. The bulk density of the sand column varied from 1.410 to $1.435 \mathrm{~g} \mathrm{~cm}^{-3}$. The experiments were conducted at $21 \pm$ $0.5^{\circ} \mathrm{C}$. Water content was measured by means of a gamma ray attenuation apparatus. The capillary pressure head was measured at six different positions in the 


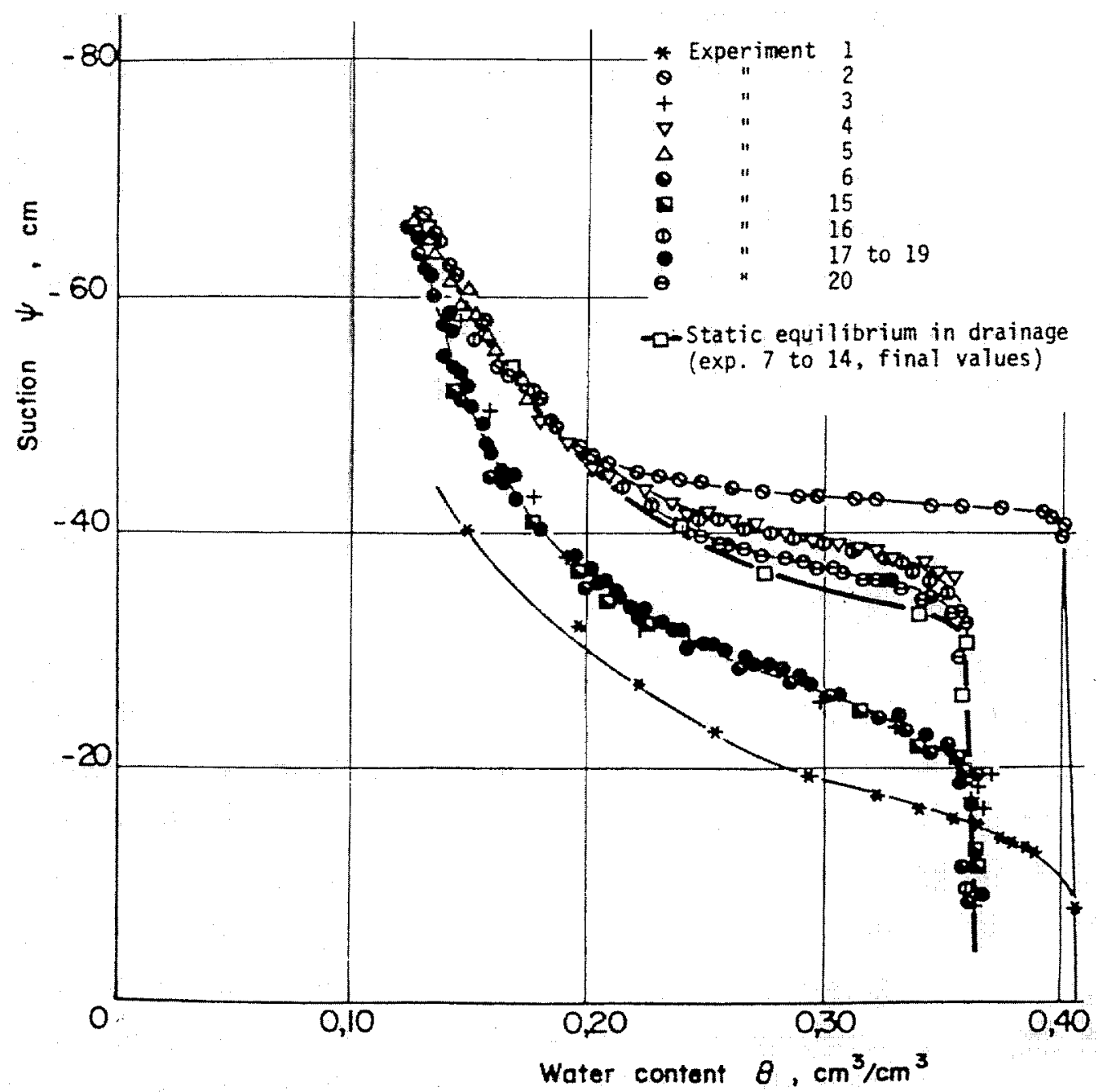

Fig. 6. Capillary pressure head-water content curves at $x=14 \mathrm{~cm}$ in experiments of Smiles et al. (1968).

column by means of 1 -cm-diam. tensiometers. The air pressure in the sample was measured and was found to have remained at atmospheric pressure at all times. The water pressure was controlled by an air-water interface at a constant height. Deaired distilled water was applied to (or allowed to leave) the column through a sintered plate. The cumulative flux into or out of the column was measured gravimetrically. All experiments were carried out on the same sand column and they consisted of cycles of wetting and drying brought about by imposing an appropriate boundary condition at the sintered plate. A large difference between the values of boundary condition and initial condition would result in a fast drainage or wetting of the column. The boundary values would eventually prevail in the whole column. Results of water content and capillary pressure head measured at a distance of $x=14 \mathrm{~cm}$ from the column inlet are shown in Fig. 6. In Step 1, the air-dry soil was saturated and this resulted in the primary wetting curve shown in Fig. 6. This curve was measured under dynamic conditions. In Step 2, $66 \mathrm{~cm}$ of suction was applied at the sintered plate, resulting in the primary drainage curve at position $x=14 \mathrm{~cm}$. Later steps were additional wetting and drainage cycles and resulted in closed loops in the $\Psi-\theta$ domain. During Steps 7 through 14 , the boundary capillary pressure head was varied incrementally from 7 to $65 \mathrm{~cm}$, with equilibrium reached in between the increments; this constitutes a quasiequilibrium drainage experiment. In Steps 17 through 19, the boundary capillary pressure head was varied gradually from 66 to $6 \mathrm{~cm}$; this forms a quasiequilibrium wetting experiment. It is evident that when a large drainage step was imposed, the measured $\Psi-\theta$ curves lay higher than the static drainage $\Psi-\theta$ curve. Smiles et al. (1971) stated that dynamic effects were not observed in the case of wetting experiments. However, we believe that the large difference between the primary wetting curve from Step 1, where the large suction in dry soil was reduced to $7 \mathrm{~cm}$ in one step, and main imbibition curve is due to dynamic effects. In this case, the dynamic wetting curve lies below the static curve. Both of these effects are in agreement with our theory.

Smiles et al. (1971) determined that the size of the dynamic effect depends on the rate at which water content changes or the size of the change in the boundary suction head. They performed additional experiments to establish that the air phase in the column was at all times at atmospheric pressure and that there was no 


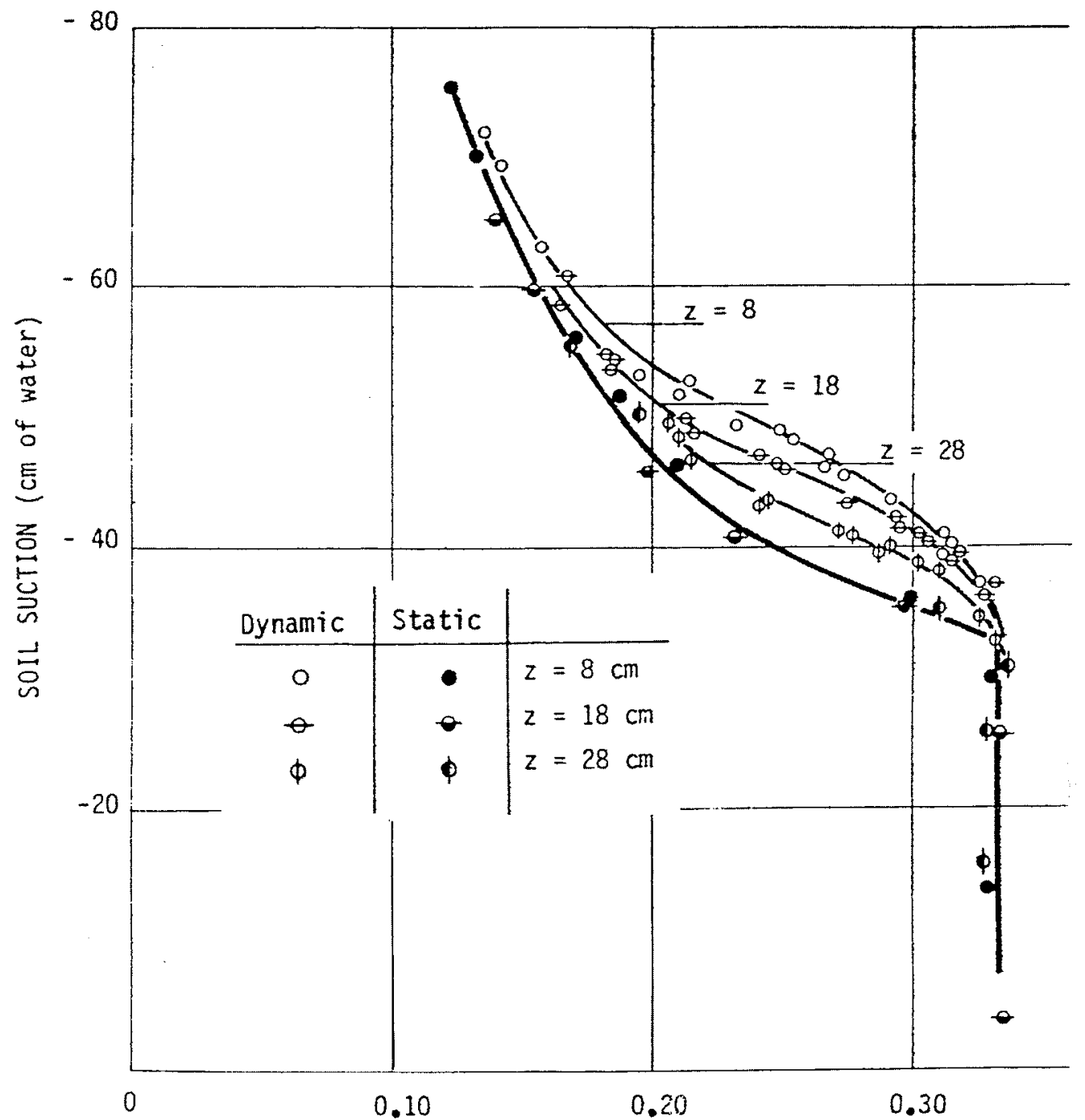

Fig. 7. Capillary pressure head-water content curves in vertical drainage experiments of Vachaud et al. (1972).

change in surface tension from a possible contamination of the water. Moreover, supplementary measurements determined that gravity had no effect on the horizontal flow assumption. The authors pointed out that the deviations between dynamic and static curves could not be due to an excessive response time in the tensiometerpressure transducer system. In fact, the result of such an effect would have been in the opposite sense. Similarly to Topp et al. (1968), they speculated that the formation and presence of isolated water pendular rings were the possible source of the dynamic effects.

Vertical drainage experiments were carried out by Vachaud et al. (1972) on a sandy soil column. The base of the column was connected to a variable piezometric surface. Initially, for all experiments, the column was saturated with the piezometric level at the top of the column $(z=0)$. Both static and dynamic drainage experiments were carried out. In static experiments, the piezometric level was lowered in increments of 5 to $10 \mathrm{~cm}$, waiting at each increment until changes in water content and pressure had ceased. In the dynamic experiment, the piezometric level was lowered suddenly from $\mathrm{z}=0$ to $\mathrm{z}=81 \mathrm{~cm}$. The air phase in the sample remained at atmospheric pressure at all times. The water content and capillary pressure were measured independently at three heights, $\mathrm{z}=8,18$, and $28 \mathrm{~cm}$, as the drainage process was proceeding. The resulting $\Psi-\theta$ curves are shown in Fig. 7. Here again, it is evident that when fast drainage occurs, the measured $\Psi-\theta$ curve lies higher than the slow (or static) curve. Vachaud et al. (1972) also found that the size of dynamic effect depends on the time rate of change in the boundary suction.

Vertical drainage experiments were also performed by Elzeftawy and Mansell (1975) on undisturbed soil cores and hand-packed columns of fine sand. They carried out static, steady-state, and transient experiments and measured both $\Psi-\theta$ and $k_{\mathrm{r}}-\theta$ curves. They obtained results similar to Topp et al. (1968). They found no significant difference between $\Psi-\theta$ data points measured at static and steady-state conditions. However, transient data points differed significantly from the static ones, showing the same trend as observed in other experiments: the larger the transient effect, the larger the difference. 


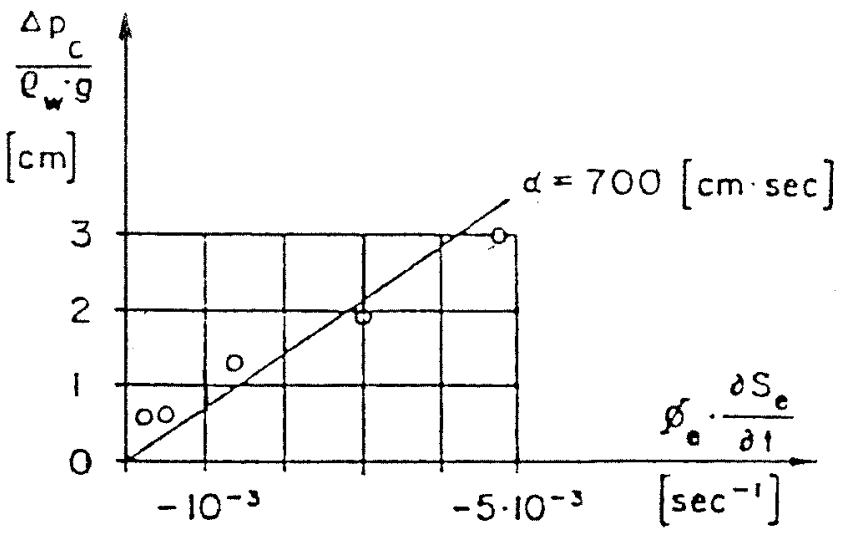

Fig. 8. Plot of the difference between dynamic and static capillary pressure head vs. time rate of change of saturation in experiments of Stauffer (1978).

Experiments similar to Vachaud et al. (1972) were carried out by Stauffer (1978). Vertical drainage experiments were performed on quartz sand packed in a Plexiglass tube. Three different columns with effective heights of 53.8, 63.8, and $16.9 \mathrm{~cm}$ were used. The grain size varied from 0.1 to $1.0 \mathrm{~mm}$, and the average porosity was about 0.335 . Water content was measured by means of a gamma ray attenuation apparatus. The suction head was measured by means of electronic transducers attached to tensiometers at five different positions in the column. The outflow rate was measured gravimetrically with the aid of a digital scale and time. The air phase pressure was also measured and was found to remain at atmospheric pressure throughout the experiments. Both quasisteady (flow at almost constant saturation) and transient experiments were carried out. The results of $P^{\mathrm{c}}-S^{\mathrm{w}}$ measurements at a location $55 \mathrm{~cm}$ above the base of the long sand column are given in Fig. 8. These results and data from similar experiments on the short column show that the transient drainage capillary pressure curves lie higher than the stationary drainage curve. Stauffer (1978) is one of the very few researchers who also examined the $k_{\mathrm{r}}-S$ relationship and found it to be nonunique as well. He quantified transient effects in $k_{\mathrm{r}}-S$ by first assuming that the relationship is given by $k_{\mathrm{r}}=S_{\mathrm{e}}^{\lambda}$, where $S_{\mathrm{e}}$ is effective saturation and $\lambda$ is a parameter. He then determined the value of $\lambda$ for steady-state and dynamic drainage experiments, and obtained the values $\lambda_{\text {st }}=$ 3.35 and $\lambda_{\text {tr }}=2.4$, respectively. This means that the relative permeability, in drainage, is higher under transient conditions than under steady-state flow. Stauffer suggested that $\lambda$ is possibly a function of $\partial s / \partial t$ but did not quantify this dependence.

A comprehensive set of experiments was carried out by Wanna-Etyem (1982). Static and dynamic curves for both drainage and imbibition experiments were performed on four different soils: dune sand, loamy sand, clay loams, and fine sand. They found dynamic effects in all cases but at varying degrees. The effects were not significant for fine-textured soil. Primary drainage curves showed more dynamic effect than the main drainage curve. In each case, the larger the rate of change of water content, the larger was the dynamic effect. This is the only experiment where the dynamic effect was clearly present in the main wetting curve as well. Again, the effect was most significant for the coarse-textured sand.

\section{Experiments Aimed at Parameter Estimation for Soil Characteristic Curves}

The question of nonuniqueness of soil characteristic curves (especially the capillary pressure-saturation curve) has received renewed attention in recent years in the framework of the analysis of one-step and multistep outflow experiments. In the standard one-step outflow method for obtaining soil characteristic curves, the water pressure at the bottom of a saturated soil column is decreased to a very low value in order to cause desaturation of the soil column. The relationship between saturation and hydraulic conductivity and capillary pressure is then established through inverse modeling of the accumulated outflow as a function of time (Kool et al., 1985). In the multistep outflow experiment, the water pressure is reduced at small steps until full desaturation of the column. A major drawback of the outflow method is known to be the dependence of resulting soil characteristic curves on the size of step in the water pressure at the lower boundary of the soil column. The nonuniqueness of results from outflow experiments has been demonstrated in a number of recent experiments by Nützmann et al. (1994), Durner et al. (1996), Hollenbeck and Jensen (1998), Schultze et al., (1999), Wildenschild and Hopmans (1999), Wildenschild et al. (2001), and Plagge et al. (1999). In all of these experiments, all drainage experiments, the dependence of soil characteristic curves on the water flow rate and/or boundary conditions was clearly demonstrated. In particular, the capillary pressure measured at a given saturation was found to be larger under transient conditions than under equilibrium conditions. Three of these experiments are described below.

Outflow experiments are basically similar to those of Vachaud et al. (1972). For example, Nützmann et al. (1994) did multiple experiments in a vertical column with a diameter of $20.6 \mathrm{~cm}$. Over a length of $150 \mathrm{~cm}$, the column was packed with a sandy soil with grain size ranging from 0.3 to $0.8 \mathrm{~mm}$. Measures were taken to obtain a homogeneous packing initially saturated with water. The column was then drained by lowering the piezometric level incrementally by a certain amount. The drop in piezometric level was increased by increments of $10 \mathrm{~cm}$ (from 20 to $60 \mathrm{~cm}$ ) or $20 \mathrm{~cm}$ (from 60 to $120 \mathrm{~cm}$ ) in subsequent experiments. Each time, the column was resaturated before the start of the next experiment. The volumetric outflow from the column was measured as a function of time. Parameters for capillary pressure-saturation curves were determined from the outflow data by means of inverse modeling. Here again, it was found that the capillary pressure curves for faster drainage lie higher than those for slower drainage.

Similar experiments were carried out by Hollenbeck and Jensen (1998) and similar results were obtained. Their sample size was much smaller $(6.35-\mathrm{cm}$ diam. and $5.7-\mathrm{cm}$ height) and the soil grain size had a bigger range $(0.18-1.65 \mathrm{~mm})$. They achieved drainage of the soil by 
Table 1. Estimates of van Genuchten parameters by Hollenbeck and Jensen (1998).

\begin{tabular}{lcccc}
\hline Parameter & Equilibrium & $\boldsymbol{\Psi}_{11,5 \rightarrow 48.5}$ & $\boldsymbol{\Psi}_{11,5 \rightarrow 68.5}$ & $\boldsymbol{\Psi}_{11.5-83.5}$ \\
\hline $\boldsymbol{\alpha}$ & $\mathbf{2 . 6 7}$ & $\mathbf{2 . 3 6}$ & $\mathbf{1 . 7 7}$ & $\mathbf{1 . 4 9}$ \\
$\boldsymbol{n}$ & $\mathbf{5 . 3}$ & $\mathbf{4 . 8 2}$ & $\mathbf{4 . 7 2}$ & $\mathbf{4 . 4 8}$ \\
\hline
\end{tabular}

increasing the air pressure. They carried out both equilibrium and transient drainage experiments. In transient experiments, the initial capillary pressure head of 11.5 $\mathrm{cm}$ was increased to $48.5,68.5$, or $83.5 \mathrm{~cm}$ in different experiments. They measured the cumulative outflow and determined parameters for capillary pressure-saturation curves by means of inverse modeling. Their estimated parameter values for van Genuchten formula are given in Table 1. These parameter values show that the transient capillary pressure curves will lie higher than the equilibrium ones; the bigger the jump in the capillary pressure head, the larger the effect will be.

Wildenschild et al. (2001) carried out single-step and multistep experiments on two types of soil: a sandy soil with $88.6 \%$ sand, $9.4 \%$ silt, and the rest clay, with a maximum grain size of $0.6 \mathrm{~mm}$, and a fine sandy loams with $63.2 \%$ sand, $27.5 \%$ silt, and the rest clay, with a maximum grain size of $0.5 \mathrm{~mm}$. They obtained both capillary pressure and relative permeability curves. They found a significant presence of dynamic effects for the sandy soil. Capillary pressure heads for fast drainage experiments were 10 to $15 \mathrm{~cm}$ higher than the slow ones. The relative permeability was up to one order of magnitude larger for the fast drainage experiments. However, they did not find any dynamics effects for the finetextured soil.

\section{ESTIMATION OF THE DYNAMIC CAPILLARY COEFFICIENT $\tau$}

The experiments described in the previous section attest to the fact that the dynamic term presented in our theory can actually be observed. Therefore, it should be possible to evaluate the material coefficient $t$ associated with this term. Equation [10] or [8] is employed for this purpose. To do so, information is needed on the difference between $P_{\mathrm{dyn}}^{\mathrm{c}}$ and $P_{\mathrm{stat}}^{\mathrm{c}}$ and on the time rate of change of saturation or water content. Some of the papers discussed above contained enough information on the experiments to allow the calculation of $\tau$. Results are given in Table 2.

The values given in this table are average values. The coefficient $\tau$ is most probably not constant and may vary with saturation. From the experiments discussed above, it appears that the size of the dynamic effect depends on soil properties such as grain size distribution, porosity, and permeability, as well as fluid properties. Stauffer (1978) suggested the following empirical relationship:

$$
\tau=\frac{\alpha \varepsilon \mu}{k \lambda}\left(\frac{p^{\mathrm{e}}}{\rho g}\right)^{2}
$$

where $\alpha$ is assumed to be constant and equal to 0.1 for all soils, $\varepsilon$ is porosity, $\mu$ is viscosity, $p^{\mathrm{e}}$ and $\lambda$ are coefficients in the Brook-Corey formula, and $k$ is the saturated permeability coefficient. This equation suggests that for fine-grained soil with a high entry pressure, $p^{\mathrm{e}}$, and a low permeability, the dynamic effect would be larger. This is indeed qualitatively observed in experiments involving wetting process (Biggar and Taylor, 1960; Nielsen et al., 1962; Rawlins and Gardner, 1963). However, in experiments involving drainage (Wildenschild et al., 2001; Wanna-Etyem, 1982), dynamic effects were absent or less significant in fine-textured soils. Thus, a phenomenological relationship for the coefficient $\tau$, in terms of medium and fluid properties, has to be developed yet.

\section{A DYNAMIC PORE-SCALE NETWORK MODEL}

In this section, a dynamic pore-scale network model is employed in order to further investigate the physical basis of dynamic capillary pressure effects. Pore-scale network models have gained popularity in recent years as a valuable tool for the study of multiphase flow and reactive solute transport in porous media (see Constantinides and Payatakes, 1996; Celia et al. 1995). In these models, the complicated pore space geometry is represented by simplified geometries wherein flow fluids and spreading of solutes can be calculated explicitly. Pore-scale network models can be used to carry out socalled numerical experiments in order to gain insight into the working and interplay of various processes and to check the validity of new theories. Pore-scale network models provide a detailed picture of flow processes within idealized pore spaces of a permeable medium. Quantities such as pressure and flow velocities are calculated at the pore scale. Corresponding macroscopic quantities are then obtained by averaging of model results. One of the advantages of pore-scale network models is their ability to give estimates of physical quantities that are impossible or very difficult to measure in labora-

Table 2. Estimates of the value of dynamic capillary coefficient $\tau$.

\begin{tabular}{|c|c|c|c|}
\hline Reference & Soil type & Type of experiment & Value of $\tau(\mathrm{kg} / \mathrm{m} . \mathrm{s})$ \\
\hline \multicolumn{4}{|l|}{ For air-water systems: } \\
\hline Topp et al. (1967) & Sand & Drainage & $\mathbf{2} \times 1 \mathbf{1 0}^{7}$ \\
\hline Smiles et al. (1971) & Sand & Drainage & $5 \times 10^{7}$ \\
\hline Elzeftawy and Mansell (1975) $\dagger$ & Fine sand & Drainage & $10^{6}$ to $10^{7}$ \\
\hline Stauffer (1978) & Sand & Drainage & $3 \times 10^{4}$ \\
\hline Wanna-Etyem (1982) & Dune sand & Drainage & $1.5 \times 10^{6}$ to $6 \times 10^{6}$ \\
\hline Nützmann et al. (1994) & Sand & Drainage & $2 \times 10^{6}$ \\
\hline Hollenbeck and Jensen (1998) & Sand & Drainage & $2 \times 10^{6}$ to $12 \times 10^{6}$ \\
\hline \multicolumn{4}{|l|}{ For oil-water systems: } \\
\hline Kalaydjian (1992b) & Sandstone/limestone & Imbibition & $\mathbf{2} \times 10^{6}$ \\
\hline
\end{tabular}

$\dagger$ Elzeftawy and Mansell (1975) provide a graph of $\partial \theta / \partial t$ where the unit is specified to be $\mathrm{cm}^{3} / \mathrm{cm}^{-3} \mathrm{~h}^{-1}$. We believe this is an error and the correct unit should be $\mathrm{cm}^{3} / \mathrm{cm}^{-3} \mathrm{~min}^{-1}$ and the values of $\tau$ given here are calculated accordingly. Otherwise, the values would be 60 times larger. 
tory experiments. In particular, fluid occupancy and pressures may be determined at any location within the idealized representation of the pore space. Thus, network models can be used to assess the significance and order of magnitude of various terms in new theories.

There are two general types of network models, quasistatic and dynamic. In quasistatic models, only equilibrium states are considered. For a given set of boundary conditions, fluid pressures are determined under hydrostatic conditions, and each fluid-fluid interface is examined for stability until a stable location is found. In this way, an equilibrium fluid distribution is found for a given set of boundary conditions. Details of movement of fluids during transition from one equilibrium state to another are not determined. In dynamic models, movements of fluids in the pore space are followed as a function of time. Fluid-fluid interfaces are tracked dynamically throughout the network. Computationally, dynamic models are much more demanding than quasistatic models. Obviously, for the study of dynamic capillary pressure effects, a dynamic pore-scale network model must be employed.

In this work, we use the dynamic network model developed by Dahle and Celia (1999). Major features of this model are described here. For more details, the article by Dahle and Celia (1999) should be consulted. In this model, the pore space is idealized as a threedimensional simple cubic lattice of spherical pore bodies and cylindrical pore throats. Although a finite radius is assigned to each pore body, they are assumed to be volumeless; all volumes are assigned to the pore throats. A cross-section of a small part of the network is depicted in Fig. 9. The fluids are assumed to be immiscible, and interfaces move via piston displacement. Given the range of pressures involved, and for computational simplicity, we assume both fluids to be incompressible. We divide time into discrete time-steps, $t^{n}, n=0,1 \ldots, N$ and solve for pressures at pore bodies $i$, for each time interval $\left(t^{n}, t^{n+1}\right)$, such that mass is conserved locally:

$$
\sum_{j \in N_{i}} Q_{i j}^{n+1}=0
$$

where $N_{i}$ denotes the set of pore bodies connected to $i$.

The flow rate through a pore throat connecting pore bodies $i$ and $j$, and containing an interface, is denoted by $q_{i j}$, and is assumed to be given by the Poiseuille formula:

$q_{i j}=\frac{\mathrm{d} l_{i j}}{\mathrm{~d} t}=-\frac{r^{2}}{8 \bar{\mu}\left(l_{i j}\right) L^{t}}\left[\bar{\rho}\left(l_{i j}\right) L g \sin \phi+\Delta p_{i j} \pm P^{\mathrm{c}}\left(r_{i j}\right)\right]$

where $l_{i j}=l_{i j}(t)$ denotes the location of a single interface within pore throat $i j$ (multiple interfaces within a pore

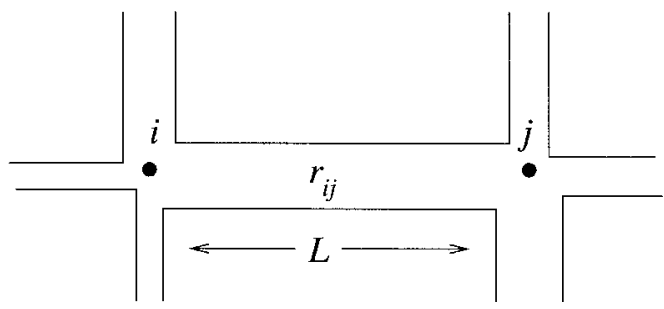

Fig. 9. Cross section of a small part of the network. throat are assumed to be trapped in this model); $L^{t}$ is the length of the throat; $\bar{\mu}$ and $\bar{\rho}$ are, respectively, average viscosity and density within the throat; $\Delta p_{i j}$ is the pressure drop across the throat. Further, $P^{\mathrm{c}}\left(r_{i j}\right)$ is microscopic capillary pressure (the pressure drop over the interface) that we assume to be given by the YoungLaplace equation (Dullien, 1992):

$$
P^{c}(R)=\frac{2 \sigma^{\mathrm{wn}} \cos \theta}{R}
$$

with $\sigma^{\text {wn }}$ being the interfacial tension, $\theta$ the contact angle, and $R$ the effective radius of the meniscus. In a cylindrical pore throat $R=r_{i j}$. Because the flow rate changes with time, the total volumetric flow in the time interval $\left(t^{n}, t^{n+1}\right)$ is given by:

$$
Q_{i j}^{n+1}=\pi r_{i j}^{2} \int_{t^{n}}^{t^{n+1}} q_{i j} \mathrm{~d} t
$$

We note that Eq. [15] and [18] constitute a nonlinear set of equations in terms of $p_{i}$ and $l_{i j}$. Furthermore, special displacement rules are required to handle trapped interfaces and also to handle the dynamics of interfaces that are transported through the junctions. These and other details related to the algorithmic development of this model are found in Dahle and Celia (1999).

To simulate a drainage experiment using the porescale network model, we assembled a three-dimensional network of size $17 \times 17 \times 29$ nodes. Dahle and Celia (1999) showed that the network is large enough so that results are practically independent of the network size. Each node represents a pore body, and six pore throats are connected to each node. Thus, the network resembled a regular Cartesian grid. The throat radii were generated from a truncated lognormal distribution specified below (see also Table 3). No attempt was made to represent the pore structure of any specific soil sample.

$$
\begin{aligned}
f\left(r ; \sigma_{\mathrm{nd}}\right)= & \frac{1}{r} \sqrt{\frac{2}{\pi \sigma_{\text {nd }}^{2}}} \exp \left[-\frac{1}{2}\left(\frac{\ln r / r_{\mathrm{ch}}}{\sigma_{\mathrm{nd}}}\right)^{2}\left[\operatorname{erf}\left(\frac{\ln r_{\mathrm{max}} / r_{\mathrm{ch}}}{\sqrt{2 \sigma_{\mathrm{nd}}^{2}}}\right)\right.\right. \\
& \left.-\operatorname{erf}\left(\frac{\ln r_{\text {min }} / r_{\mathrm{ch}}}{\sqrt{2 \sigma_{\text {nd }}^{2}}}\right)\right]^{-1}
\end{aligned}
$$

Initially, the network was completely filled with wetting fluid connected to a reservoir at the bottom boundary at a given pressure $p^{\mathrm{B}}=0 \mathrm{~Pa}$. The top boundary was connected to a reservoir filled with nonwetting fluid at a given pressure $p^{\mathrm{T}}$. Pore throats connected to the four vertical boundaries were assumed to have dead ends (no-flow condition). The pressure at the top boundary was increased by a specified pressure step $\Delta p$ until the nonwetting fluid started to invade the network. After that $p^{\mathrm{T}}$ was increased by $\Delta p$ each time the fluid distribution reached an equilibrium configuration. A mov-

Table 3. Parameters for network and drainage experiment.

\begin{tabular}{lll}
\hline Contact angle & $\boldsymbol{\theta}$ & $0^{\circ}$ \\
Surface tension & $\boldsymbol{\sigma}^{\mathrm{wn}}$ & $\mathbf{7 . 2} \times \mathbf{1 0}^{-2}[\mathrm{~N} / \mathrm{m}]$ \\
Viscosity-wetting phase & $\boldsymbol{\mu}_{w}$ & $\mathbf{1 0}^{-3}\left[\mathrm{Ns} / \mathbf{m}^{2}\right]$ \\
Viscosity-non wetting phase & $\boldsymbol{\mu}_{n w}$ & $\mathbf{1 0}\left[\mathrm{Ns} / \mathbf{m}^{2}\right]$ \\
Length of tubes & $\boldsymbol{L}$ & $\mathbf{0 . 3 3 2}[\mathbf{m m}]$ \\
Lower cut-off radius & $\boldsymbol{r}_{\min }$ & $\mathbf{1}[\mathbf{m m}]$ \\
Upper cut-off radius & $\boldsymbol{r}_{\max }$ & $\mathbf{4 0}[\boldsymbol{\mu m}]$ \\
Characteristic radius & $\boldsymbol{r}_{c h}$ & $\mathbf{1 0}[\boldsymbol{\mu m}]$ \\
Standard deviation, parent distribution & $\boldsymbol{\sigma}_{n d}$ & $\mathbf{0 . 1}$ \\
\hline
\end{tabular}




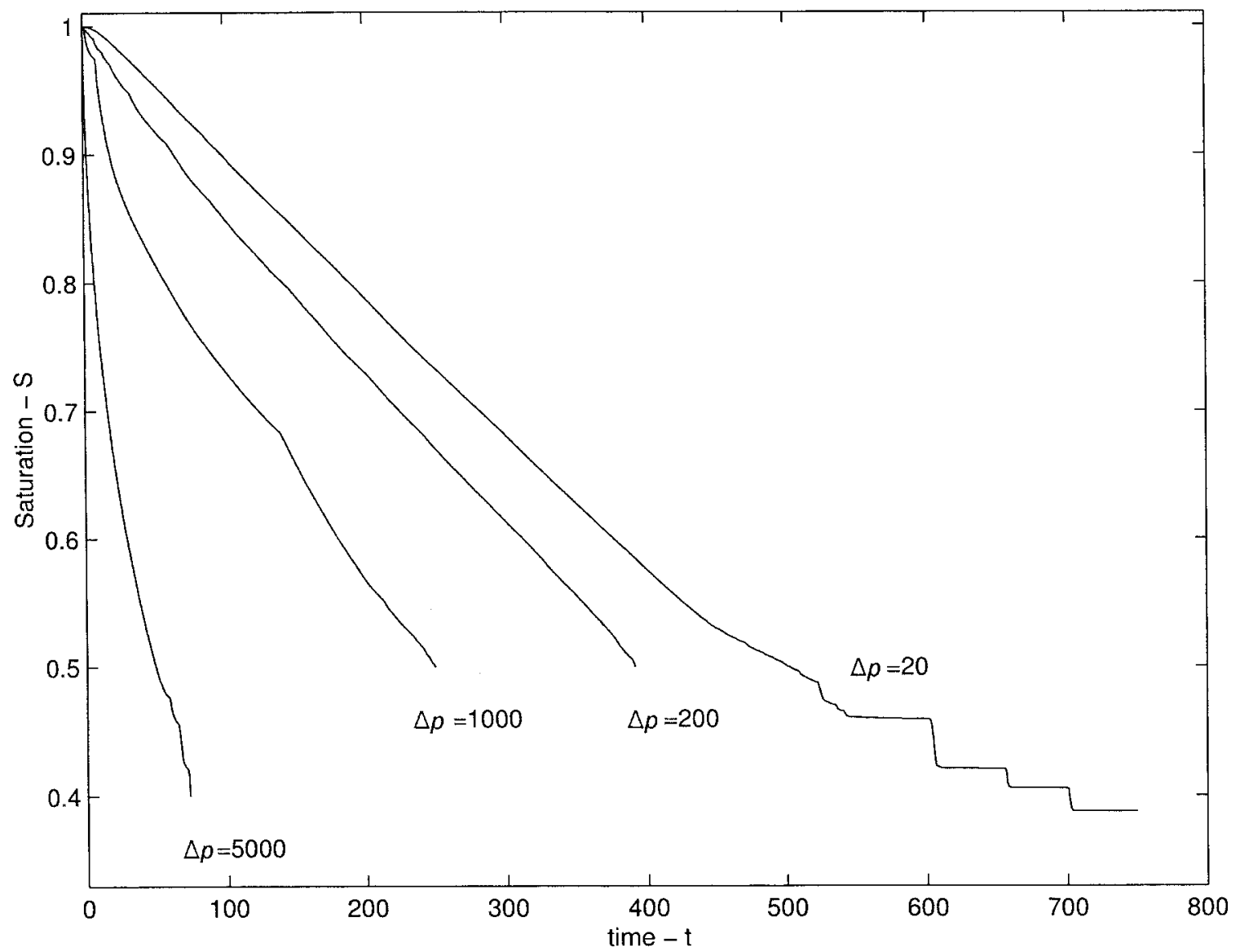

Fig. 10. Saturation vs. time (seconds). $\Delta p=20,200,1000$, and $5000 \mathrm{~Pa}$.

ing average of the saturation change per time, $|\Delta \bar{S} / \Delta t|$, was successively calculated for a window of ten time steps. Saturation is calculated for the whole network on the basis of the fluid distributions within pore throats. The equilibrium condition was set to be $|\Delta \bar{S} / \Delta t|<10^{-3}$ $(1 / \mathrm{s})$. A simulation was run until a residual saturation of the wetting fluid was reached. The results reported here are based on four simulations, with $\Delta p=20,200$, 1000 , and $5000 \mathrm{~Pa}$, respectively. Gravity effects were neglected in these simulations.

For each set of simulations, we calculated the saturations $S=S(t)$ at each time-step $t^{n}$ as shown in Fig. 10. A natural definition of the macroscopic capillary pressure is given by the pressure difference associated with the boundary fluid reservoirs,

$$
P^{\mathrm{c}}(S)=p^{\mathrm{T}}-p^{\mathrm{B}}=p^{\mathrm{T}}
$$

To be more consistent with the thermodynamic averaging theory of Hassanizadeh and Gray (1990), we also computed the volume-averaged phase pressures at each time step:

$$
\bar{p}_{\alpha}(t)=\frac{\iiint_{V} p_{\alpha}(x, t) \gamma_{\alpha}(x, t) \mathrm{d} x}{\iiint_{V} \gamma_{\alpha}(x, t) \mathrm{dx}} \quad \alpha=w, n w
$$

Here, integration is over the throats in the network and the indicator function $\gamma_{\alpha}$ is 1 if phase $\alpha$ is present and 0 otherwise. Based on average phase pressures (Eq. [18]), an alternative capillary pressure function is de- fined by:

$$
\bar{P}^{\mathrm{c}}[S(t)]=\bar{p}_{\mathrm{nw}}(t)-\bar{p}_{\mathrm{w}}(t)
$$

Similar to Eq. [19], Eq. [21] may be defined for any given saturation, and may be viewed as a dynamic definition of the $P^{\mathrm{c}}-S$ relationship away from the equilibrium points. Again this is to be used to estimate the dynamic coefficient $\tau$ in Eq. [7]. Drainage curves based on the definition in Eq. [21] are plotted in Fig. 13. Observe that the equilibrium points are virtually on top of each other for large saturations, whereas transient curves (generated by large $\Delta p$ values) lie higher. In particular, this is evident for the cases where $\Delta p=5000$ and 1000 $\mathrm{Pa}$. Note that the local pressure distribution is dictated by the boundary pressures and the pressure drops over the fluid-fluid interfaces. If ganglia of fluids that have become disconnected from their respective reservoirs constitute a significant portion of the averaging volume, then nonmonotonic capillary pressure curves may result. This can be seen in Fig. 13 in the case of large $\Delta p$ and small saturations. When any of these is flushed out of the network by the flowing phase, sudden changes in the average phase pressure can result.

We believe that results of cases $\Delta p=20$ and $200 \mathrm{~Pa}$ are close to equilibrium (static situation), whereas $\Delta p=$ 1000 and $5000 \mathrm{~Pa}$ represent a dynamic situation. At least, this seems to be the situation as long as outflow boundary effects can be ignored. This observation will be used below when we estimate $\tau$. 


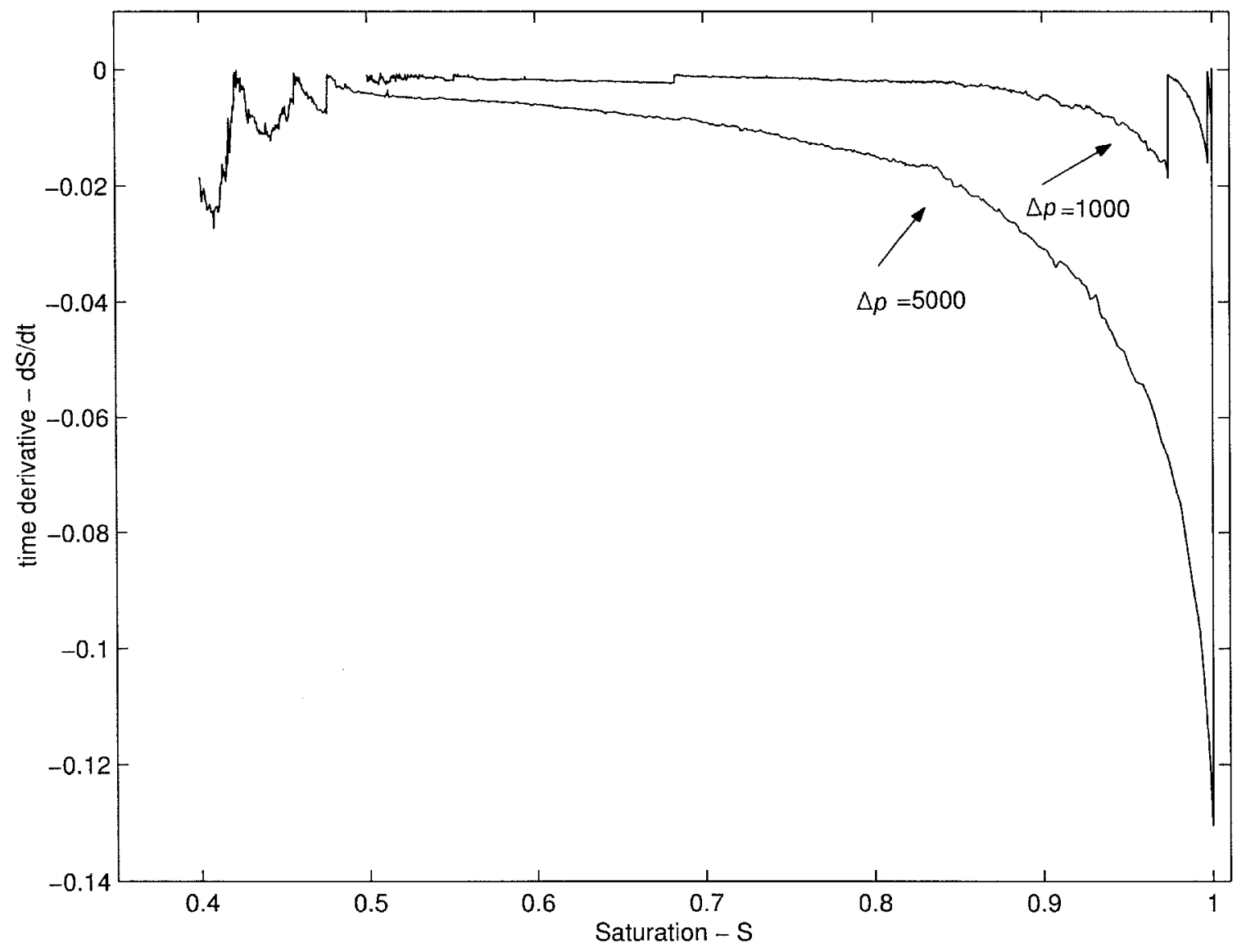

Fig. 11. Time derivative of saturation vs. saturation at $\Delta p=1000$ and $5000 \mathrm{~Pa}$.

The time-derivative of the saturation can be calculated from the curves in Fig. 10. Results are shown in Fig. 11 for $\Delta p=1000$ and $5000 \mathrm{~Pa}$. To avoid noise in the derivatives for $\Delta p=20$ and $200 \mathrm{~Pa}$ (not shown), we produced least square linear fits to the saturation curves to obtain "average" time derivatives.

We may apply Eq. [7] to two different $P^{\mathrm{c}}-S$ curves (e.g., for $\Delta p_{1}=200 \mathrm{~Pa}$ and $\Delta p_{2}=5000 \mathrm{~Pa}$ ) and subtract them from each other to eliminate $P_{\text {stat }}^{\mathrm{c}}$. We obtain:

$$
P_{1}^{\mathrm{c}}-P_{2}^{\mathrm{c}}=-\tau\left(\mathrm{d} S_{1} / \mathrm{d} t-\mathrm{d} S_{2} / \mathrm{d} t\right)
$$

We then calculate data points

$$
[x(S), y(S)]=\left(P_{1}^{\mathrm{c}}-P_{2}^{\mathrm{c}}, \mathrm{d} S_{1} / \mathrm{d} t-\mathrm{d} S_{2} / \mathrm{d} t\right)
$$

Here, subscript 1 refers to a low- $\Delta p$ simulation $\left(\Delta p_{1}=\right.$ 20 or 200) and subscript 2 to a high- $\Delta p$ simulation $\left(\Delta p_{2}=\right.$ 1000 or 5000 ).

By assuming that $\tau$ is independent of saturation $S$, we can obtain estimates for $\tau$ as the slope of a least square linear fit to all the data points $(x, y)$ in Eq. [23]. However, the spread of these data points did not make this very meaningful, and possibly suggests that $\tau$ depends on saturation $\tau=\tau(S)$.

On the other hand, consider the intervals between the equilibrium points for $\Delta p_{2}=1000$ and 5000, shown on Fig. 12. These intervals can also be identified from Fig. 11 and 13. To avoid boundary effects we look at the four intervals found between $S \approx 0.55$ and $S=1$ for $\Delta p_{2}=1000$, and the single interval between $S \approx 0.48$ and $S=1$ for $\Delta p_{2}=5000$. Within each such interval, a least square linear fit gave a fairly good approximation to the data points. A typical example of the fit is shown for one interval in Fig. 14. Estimated values of $\tau$, using both definitions for the $P^{\mathrm{c}}-S$ curves (i.e., Eq. [19] and [21]) are given in Table 4 . The values vary between $2.10^{4}$ and $2.10^{5} \mathrm{~kg}(\mathrm{~m} \mathrm{~s})^{-1}$.

\section{INCLUSION OF DYNAMIC $P^{\mathrm{c}}$ IN UNSATURATED FLOW SIMULATORS}

\section{Governing Equation and Numerical Approximations}

Standard unsaturated flow simulators solve Eq. [9] numerically using various discretization methods (see, e.g., Celia et al., 1990). In those simulators, the water pressure is assumed to be a prescribed function of moisture content, and a single equation is solved. If dynamic capillary pressure is to be included in simulators, then Eq. [9] must be solved with water pressure head interpreted properly. Therefore, an additional equation, namely Eq. [8], must be incorporated into the simulator.

At least two options may be pursued to solve Eq. [8] and [9] numerically. The first involves iterative solution of the coupled set of two equations. For example, one may substitute the expression for $\partial \theta / \partial t$ from Eq. [8] into Eq. [9]. This creates a kind of elliptic equation for which, given an estimate of moisture content, $\Psi_{\mathrm{dyn}}$ may be 


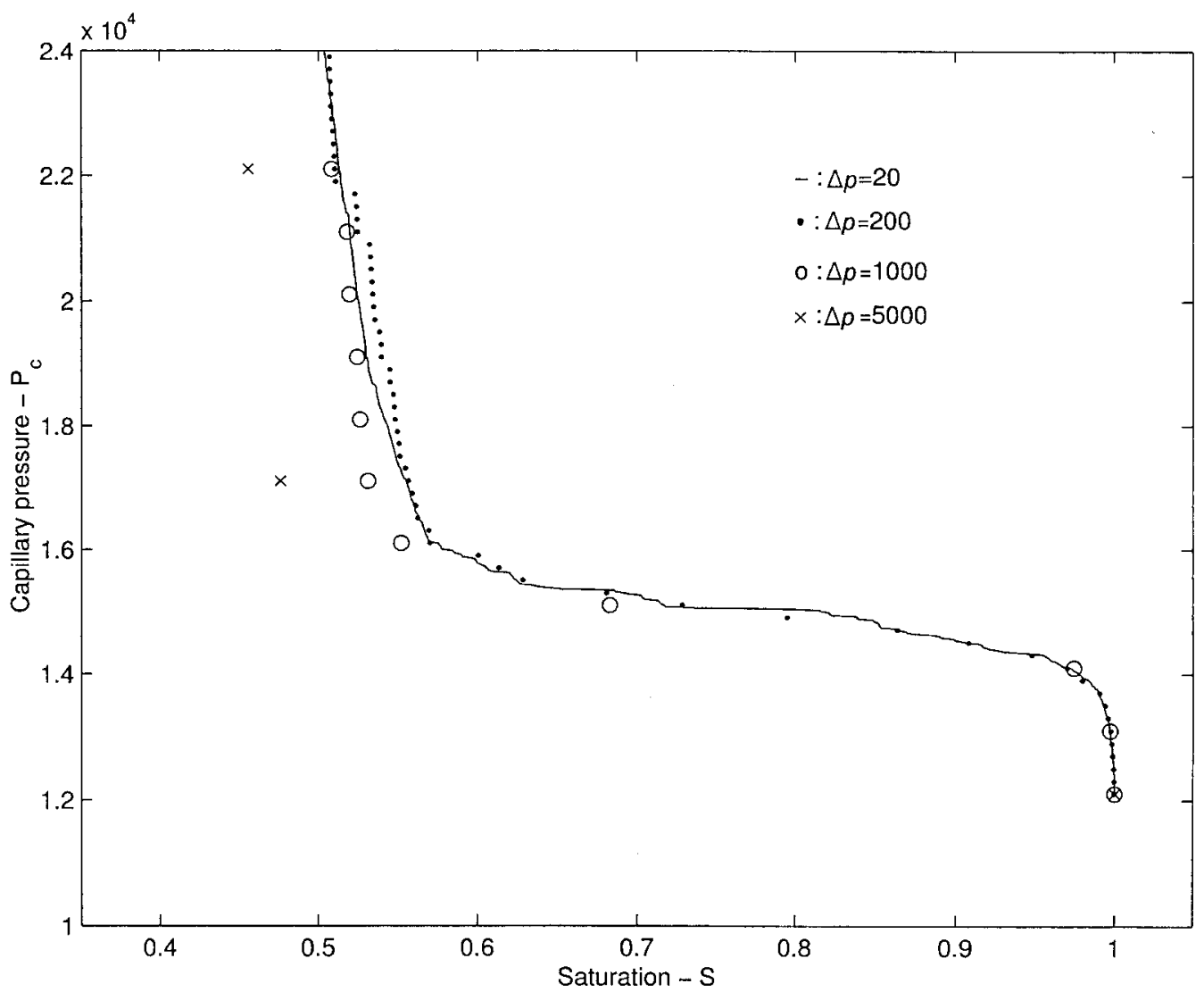

Fig. 12. Primary drainage curve using Eq. [19].

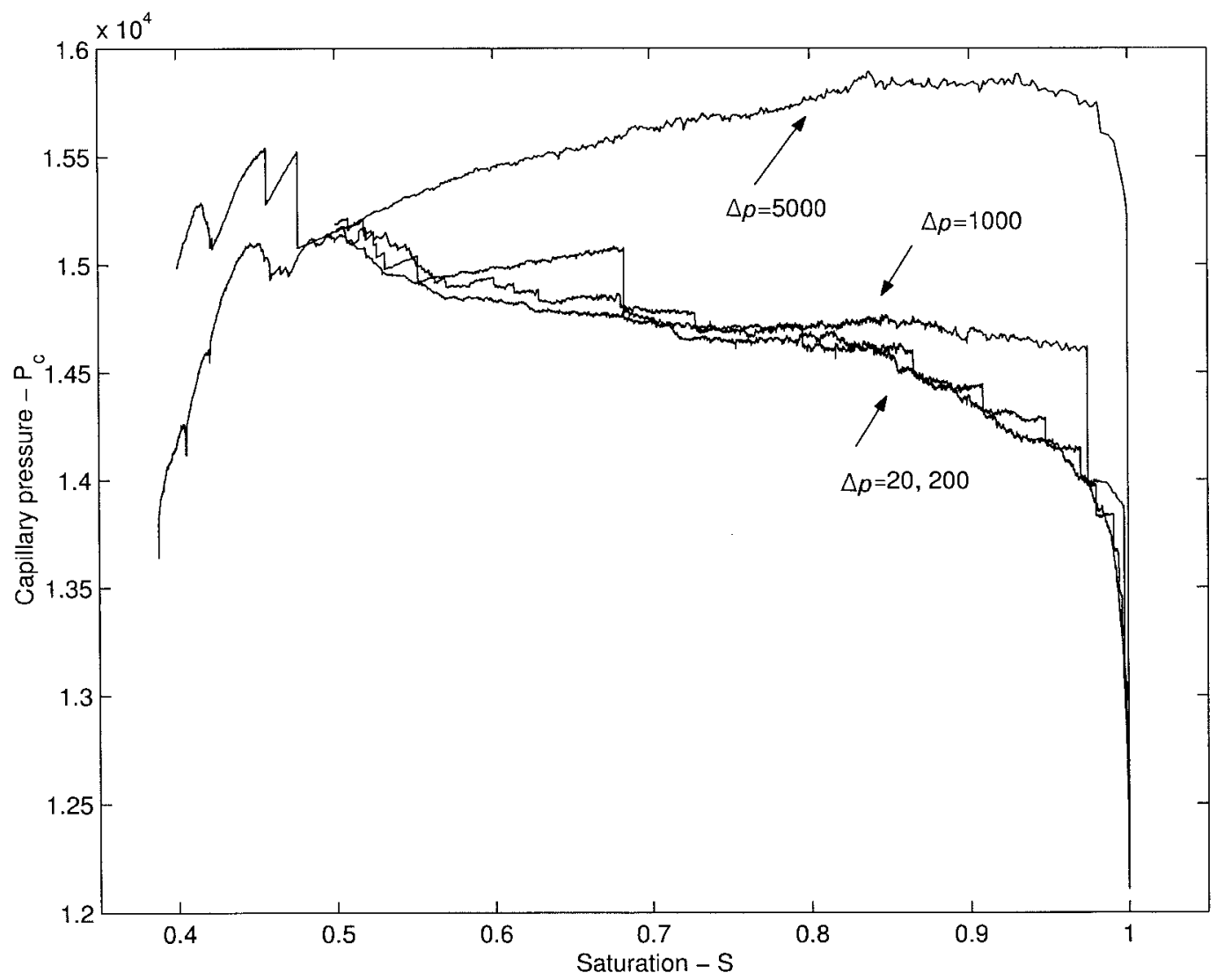

Fig. 13. Primary drainage curves using Eq. [21]. 

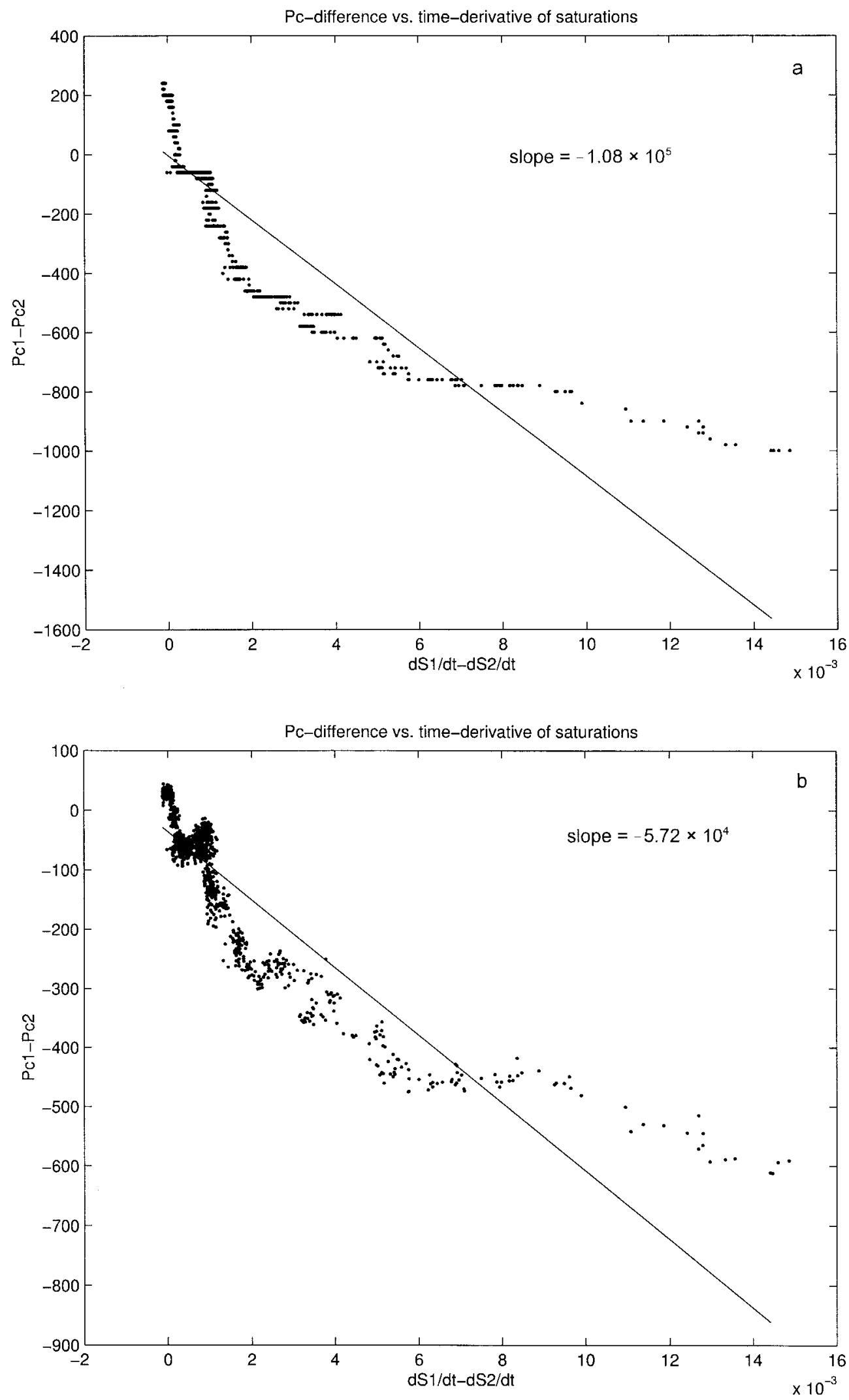

Fig. 14. Estimate of $\tau$ for $\Delta p_{1}=20 \mathrm{~Pa}, \Delta p_{2}=1000 \mathrm{~Pa}$, and $0.67<S<0.97$. Dynamic $P^{\mathrm{c}}$ is given by Eq. [19] in (a) and by Eq. [21] in (b). 
Table 4. Estimate of dynamic coefficient $\tau$.

\begin{tabular}{|c|c|c|c|c|}
\hline \multicolumn{2}{|c|}{$\begin{array}{c}\text { Pressure } \\
\text { increments (Pa) }\end{array}$} & \multirow{2}{*}{$\begin{array}{c}\text { Saturation } \\
\text { range }\end{array}$} & \multicolumn{2}{|c|}{$\tau \times 10^{-4}(\mathrm{~kg} / \mathrm{m}-\mathrm{s})$} \\
\hline$\Delta P_{1}$ & $\Delta P_{2}$ & & $\bar{P}^{c}$ (Eq. [21]) & $P^{c}$ (Eq. [19]) \\
\hline \multirow[t]{4}{*}{20} & 1000 & {$[0.997,0.999]$} & 5.97 & 7.06 \\
\hline & & {$[0.975,0.995]$} & 3.98 & 6.46 \\
\hline & & {$[0.68,0.97]$} & 5.72 & 10.8 \\
\hline & & {$[0.55,0.68]$} & 24.6 & 144.0 \\
\hline 20 & 5000 & {$[0.48,0.99]$} & 3.06 & 15.6 \\
\hline \multirow[t]{4}{*}{200} & 1000 & {$[0.997,0.999]$} & 7.09 & 7.66 \\
\hline & & {$[0.975,0.995]$} & 3.14 & 5.04 \\
\hline & & {$[0.68,0.97]$} & 5.54 & 10.1 \\
\hline & & {$[0.55,0.68]$} & 25.1 & 153.0 \\
\hline 200 & 5000 & {$[0.48,0.99]$} & 2.65 & 8.19 \\
\hline
\end{tabular}

solved. Equation [8] may be used to update moisture content, and an iterative procedure is then followed (see Beliaev and Hassanizadeh, 2001). While this procedure follows a natural iterative strategy, it can only be used when the coefficient $\tau$ is nonzero. In addition, it involves modification to the structure of existing Richards' equation simulators. We have implemented this approach, and report results below, but we are also motivated to seek an alternative formulation that allows existing codes to be modified with minimal effort, and that also accommodates solution to the traditional Richards' equation (i.e., cases when $\tau=0$ ).

One alternative approach involves rearrangement of Eq. [8] to express dynamic pressure in terms of moisture content and static pressure,

$$
\Psi_{\mathrm{dyn}}=\Psi_{\text {stat }}+\frac{\tau}{\varepsilon \rho g}\left(\frac{\partial \theta}{\partial t}\right)
$$

This expression may then be substituted into Eq. [9] to produce an equation in which only moisture content $\theta$ and static pressure head $\Psi_{\text {stat }}$ appear. The resulting equation also has the form of the usual Richards' equation, with the exception of an additional term. That additional term is a third-order mixed derivative.

$$
\frac{\partial \theta}{\partial t}-\frac{\partial}{\partial x}\left[k_{\mathrm{r}}(\theta) K \frac{\partial \Psi_{\text {stat }}}{\partial x}\right]-\frac{\partial}{\partial x}\left[k_{\mathrm{r}}(\theta) K \frac{\partial}{\partial x}\left(\hat{\tau} \frac{\partial \theta}{\partial t}\right)\right]
$$

The additional term, with $\hat{\tau}=\tau / \varepsilon \rho g$, involves two spatial derivatives and one temporal derivative. As such, its order is consistent with the other terms in the equation. This means that any finite difference or finite element approximation will involve the same computational footprint as for the standard Richards' equation. Therefore, as a first approach to solving this equation numerically, standard numerical procedures for Richards' equation may be adapted to include values associated with this extra term, with relatively little effort. This approach is followed herein, where the Modified Picard approach of Celia et al. (1990) is expanded to include the extra terms derived from the dynamic capillary pressure. For static systems, the coefficient $\tau=0$, and the approximation reverts to the standard Richards' approximation. Notice that if the flow solution is used to calculate water velocities, then the dynamic pressure must be recovered through a post-processing step involving Eq. [8]. However, this is external to the iterative solution procedure.
Saturation Profiles at $\mathrm{t}=\mathbf{1 2} \mathrm{h}$

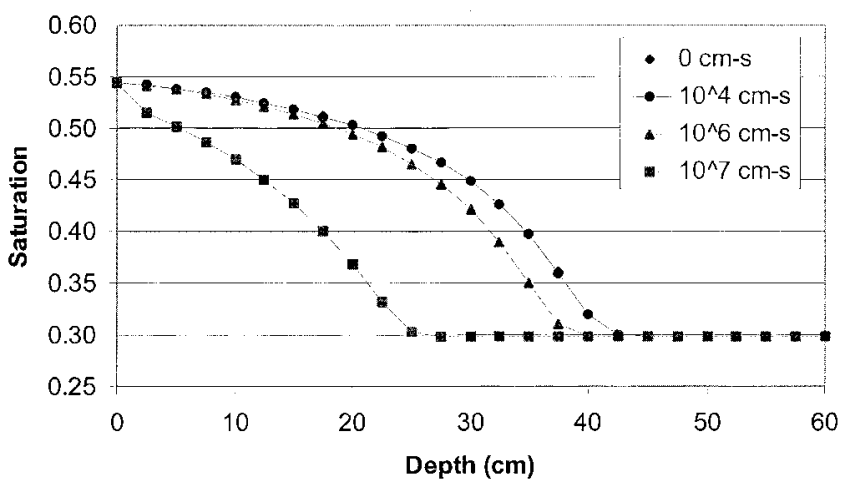

Fig. 15. Saturation profiles at time $t=12 \mathrm{~h}$, for various values of $\tau$.

\section{Example Calculations}

To examine the impact of measured values of the dynamic coefficient $\tau$ on unsaturated flow systems, we consider a simple example of infiltration into a relatively dry soil. This problem was solved by Celia et al. (1990), and is based on field data collected at a location in New Mexico, USA. A range of dynamic coefficients is used, based on the values given in Tables 2 and 4. These coefficients range from $10^{4}$ to $10^{7} \mathrm{~kg}(\mathrm{~m} \mathrm{~s})^{-1}$. Given a density of $1000 \mathrm{~kg} \mathrm{~m}^{-3}$, and a value of gravitation acceleration of $9.8 \mathrm{~m} \mathrm{~s}^{-2}$, the range of modified coefficient $\hat{\tau}$ is of the order of $10^{0}$ to $10^{4} \mathrm{~m} \mathrm{~s}$, or $10^{2}$ to $10^{6}$ $\mathrm{cm} \mathrm{s}$. The example problem uses the van Genuchten functional forms to describe the soil properties, with values as follows: $\alpha=0.0335, \theta_{\mathrm{s}}=0.368, \theta_{\mathrm{r}}=0.102$, $n=2$, and $K_{\text {sat }}=0.00922 \mathrm{~cm} \mathrm{~s}^{-1}$. Initial conditions are taken as a uniform suction head of $-1000 \mathrm{~cm}$, the bottom boundary is held at that initial head, and the top boundary head is fixed at $-75 \mathrm{~cm}$. Because the moisture content at the boundaries is not changing with time, the dynamic pressure is equal to the static pressure, and the assigned boundary pressures are unambiguous.

Results are shown in Fig. 15, where saturation $S$ is plotted as a function of depth, at a time of $12 \mathrm{~h}$. Four different infiltration fronts are shown, corresponding to values of $\hat{\tau}$ equal to $0,10^{4}, 10^{6}$, and $10^{7} \mathrm{~cm} \mathrm{~s}$. The first value represents the purely static case, the second represents a values intermediate to the experimentally observed range, the third value represents the largest measured values reported, and the fourth value represents one order of magnitude greater than the largest reported measured value. The figure shows that for this soil, values of $\hat{\tau}<10^{6} \mathrm{~cm}$ s have little effect on the infiltration front. When $\hat{\tau}=10^{6} \mathrm{~cm} \mathrm{~s}$, the infiltration depth is modified by about $10 \%$. However, for the case of $\hat{\tau}=10$ $\mathrm{cm} \mathrm{s}$, a pronounced modification is seen, with the front retarded by about $50 \%$. Similar behavior is observed in the dynamic pressure front, which is plotted for these same simulations in Fig. 16. These results demonstrate the range of coefficients for which this particular soil is sensitive to dynamic capillary pressure effects. We expect results to differ for different soils, and expect certain dimensionless groups or other parameters to provide a systematic characterization of the importance of dynamic effects for particular soils. In addition, the problem solved here involved fixed-head boundary con- 


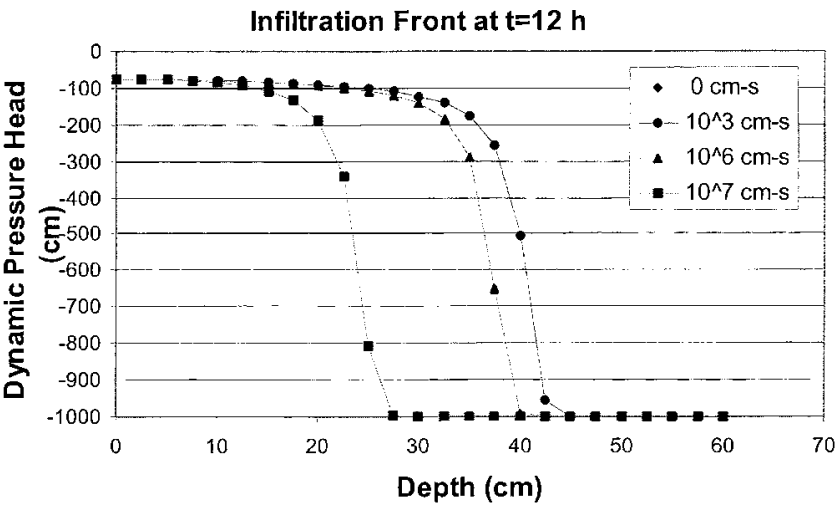

Fig. 16. Calculated dynamic pressure head profiles for several values of $\tau$ at $t=12 \mathrm{~h}$.

ditions. More general conditions, including flux conditions and temporal and spatial variabilities remain to be explored.

\section{DISCUSSION AND CONCLUSIONS}

We have presented a nonequilibrium equation for capillary pressure that is valid under transient conditions. The experiments reviewed here provide strong support for the ability of this equation to model transient unsaturated flow processes. Some of these papers contain enough data to allow the calculation of the dynamic coefficient $\tau$. We have found that this coefficient varies between $10^{4}$ and $10^{7} \mathrm{~kg}(\mathrm{~m} \mathrm{~s})^{-1}$.

The fact that the capillary pressure-saturation relationship is not unique has been known for quite some time. However, a theoretical framework for the inclusion of these deviations in the theories of multiphase flow in a systematic manner has been lacking. Vachaud et al. (1972) plotted the capillary head $\Psi$ as a function of $\partial \Psi / \partial t$, implying a functional relationship between the two. However, the relationship seems to be nonlinear and they did not propose any specific formula. Plots of $\partial \Psi / \partial t$ vs. $\Psi$ were also made by Elzeftawy and Mansell (1975). These were highly nonlinear. These results were interpreted by Watson and Whisler (1978) to mean that in vertical drainage the $\Psi-\theta$ relationship is space-dependent. They assumed that the parameters in the BrooksCorey relationship are functions of depth and carried numerical simulation for hypothetical situations similar to those in the experiments by Vachaud et al. (1972) and Elzeftawy and Mansell (1975). Their calculated plots of $\partial \Psi / \partial t$ vs. $\Psi$ showed the same characteristics as the measurements from those experiments.

Stauffer (1978) plotted the deviation of dynamic capillary head from static values, $\Delta \Psi$, as a function of $\partial \Psi / \partial t$ and found no obvious relationship. He also plotted $\Delta \Psi$ as a function of $\partial S / \partial t$ and found a reasonably linear relationship between the two. From these results and merely on an empirical basis, he proposed the use of a formula similar to Eq. [7]. A more rigorous derivation of Eq. [7] was achieved by Kalaydjian (1992a), who employed a procedure based on volume-averaging methods and thermodynamics considerations. The approach of Hassanizadeh and Gray (1990), described above, was based on the application of the second law of thermodynamic and led to the derivation of Eq. [6].

As explained above, the coefficient $\tau$ behaves as a capillary damping coefficient. In an attempt to produce a damping effect (or in their words, to account for nonequilibrium effects), Barenblatt and Gil'man (1987) introduced what they called "effective water saturation" $\eta$. This effective saturation was related to the real saturation by:

$$
\eta=\mathrm{S}+\tau * \frac{\partial S}{\partial t}
$$

where $\tau^{*}$ is a constant referred to as "substitutional time." The relative permeability coefficients and capillary pressure were assumed to be functions of $\eta$ instead of $S$. Obviously, under equilibrium conditions, the distinction between the two saturations disappears. This is a purely empirical approach wherein Eq. [26] is introduced in order to induce a desired mathematical behavior in equations of two-phase flow. In the context of this approach, the capillary pressure is the same under static and dynamic conditions, but the saturation will be different. It is not clear how parameters $\eta$ and $\tau^{*}$ are to be measured. Even if inverse modeling is used to estimates these parameters, their physical meaning remains obscure.

Our survey of experiments performed during the last five decades clearly shows that dynamic effects are significant for air-water systems in drainage as well as imbibition. Dynamic effects are observed not only in the capillary pressure-saturation curve but also in the relative permeability-saturation curve. However, the number of experiments addressing the latter effect is limited. The underlying mechanisms of the dynamic effects are yet to be identified with certainty. Various authors have mentioned different causes. As summarized by Wildenschild et al. (2001), major potential mechanisms suggested in the literature are considered to be:

1. Water entrapment - this can be the case for drainage at high flow rate. Due to rapid drainage of larger pores, water in smaller pores can become hydraulically isolated and thus result in a larger saturation under dynamic conditions. Under such conditions, the relative permeability is expected to be lower, as the mobile portion of the soil water is decreased. This is, however, in contrast with the results of Wildenschild et al. (2001), where the relative permeability was found to be larger under dynamic conditions at high saturations.

2. Pore water blockage near the bottom (drainage) boundary - this can be the case when a sudden large pressure gradient is applied to drain a soil sample. The reasoning here is that the pores near the bottom boundary drain quickly and, assuming continuity of air phase throughout the sample, are filled with air. This causes water to stay behind. But, here again, this effect should reduce the overall relative permeability. That is not found to be the case. Also, this effect will not necessarily occur under field conditions.

3. Air entrapment - this can be the case for drainage at high saturation. The water can be drained only if air can replace it. But if the air phase is not 
continuous, which can occur in a sample holder that is open to air only at the top, the water cannot be drained effectively and this leads to a higher saturation under dynamic conditions. This effect is not expected to occur under field conditions.

4. Dynamic contact angle effect-this has been suggested by Aribert (1970) and by Friedman (1999). From experiments in single capillaries, it is known, as compared with a static position, that the radius of curvature of fluid-fluid interfaces decreases during drainage (i.e., a smaller contact angle) and increases during imbibition (i.e., a larger contact angle) (see Hillel [1980] or Lake [1989] and references therein). Compared with quasistatic conditions, this phenomenon could result in a higher capillary pressure for drainage and a lower capillary pressure for imbibition under dynamic conditions. This effect can be significant for drainage only if the static contact angle is rather large (e.g., in oil-water systems) and for imbibition only if static contact angle is rather small (e.g., in airwater systems).

The first three of the above effects are relevant for drainage processes only, whereas dynamic effects have been observed for imbibition as well. Moreover, these nonequilibrium mechanisms all occur at the pore scale. We believe that even if there is equilibrium at the pore scale, the upscaling, in this case the volume averaging, of complex pore-scale processes will always introduce nonequilibrium effects. In addition to pore-scale complexities, we believe that the presence of microheterogeneities (e.g. subsample-scale variations in the packing of the soil sample) also results in nonequilibrium effects in observations made at the sample scale. In any case, and regardless of the underlying mechanisms, such nonequilibrium effects manifest themselves in the form of a relaxation term. Our proposed dynamic capillary pressure equation properly introduces such a relaxation effect into the two-phase theory. Additional research is still needed to clarify the underlying mechanisms of these dynamic effects.

The work reported herein is not only based on sound theoretical underpinnings, but it integrates two different types of numerical models. These models include a dynamic pore-scale network model, used to estimate the dynamic coefficient $\tau$, and two continuum-scale models, both of which are meant to provide initial estimates of the practical effects of dynamic capillary pressure. The network model offers the ability to perform mathematical experiments over a wide range of material types and microheterogeneity patterns. As such, dynamic effects may be investigated systematically over wide ranges of conditions, and the range of dynamic coefficients can be defined much more fully. These models represent a critical tool that can provide important information about coefficients defined on the core scale. These coefficients can then be used in continuum-scale simulators to begin to explore the possible practical implications of dynamic capillary pressure for field problems.

The continuum-scale simulators developed herein are relatively simple in their construction and their numerical structure. Both are based on cell-centered finite differences, and both use relatively simple iteration schemes.
They differ in their numerical behavior in several important ways. First, we note that the single-equation approach is attractive because it requires little modification to existing codes, and it inherently accommodates the case of $\hat{\tau}=0$. However, for the largest values of $\hat{\tau}$, the current implementation of the numerical algorithm fails to converge. This appears to be related to the mixed-order derivative and its effect on the matrix coefficients in the discrete equations. Our investigations of this phenomenon are ongoing. The two-equation algorithm converges well for larger values of $\hat{\tau}$, so this algorithm was used to compute solutions for $\hat{\tau}$ of $10^{6}$ and $10^{7} \mathrm{~cm} \mathrm{~s}$. For intermediate values of $\hat{\tau}$, the two algorithms produce nearly identical solutions, so in that range, the algorithms are essentially interchangeable. While we are studying the convergence properties of the singleequation algorithm, we are also investigating possible hybrid approaches that use a single-equation for small values of $\hat{\tau}$, while the two-equation approach is used for large values of $\hat{\tau}$.

Finally, we comment on the range of coefficients considered, and the effects they have on the solutions. Given that the largest measured values for $\hat{\tau}$ are around $10^{6} \mathrm{~cm} \mathrm{~s}$, the calculations for the single infiltration scenario considered herein indicate that dynamic capillary pressure has a small effect on the overall solutions for this problem. However, we also see that the value of $10^{6} \mathrm{~cm} \mathrm{~s}$ is just at the boundary of where significant effects are apparent, and an increase of one more order of magnitude produces pronounced changes in the infiltration profiles. While additional experimental studies are needed to determine if conditions exist for which larger values of $\hat{\tau}$ are produced, we expect that certain practical situations can lead to larger values for this coefficient. For example, systems involving heterogeneous media, for which upscaled pressures and saturations are used in the governing equations, are expected to have amplified dynamic capillary pressure effects, with concomitant retardation of infiltration fronts. We are in the process of studying such systems using computational tools, and hope to report on the relationship between upscaling and dynamic pressure effects in future publications. For now, we can report that initial simulations indicate ranges for which dynamic effects may be important. These begin to provide an initial framework for systematic study of this potentially important phenomenon.

\section{ACKNOWLEDGMENTS}

The senior author is grateful to John L. Nieber of University of Minnesota for fruitful discussions on this subject and for bringing the experiments in soil physics literature to his attention. The authors appreciate stimulating interactions with William G. Gray of University of Notre Dame during our longstanding collaboration. Collaboration between $\mathrm{SMH}$ and MAC has been facilitated through a Visiting Faculty appointment provided to M.A. Celia by Delft University of Technology. Various parts of this research have been carried out in the framework of project no. ALW 809.62.012 financed by the Dutch Organization for Scientific Research and project no. 121509/410 financed by the Norwegian Research Council. Comments by two anonymous referees are gratefully acknowledged. 


\section{REFERENCES}

Aribert, J.M. 1970. Problèmes de déplacement d'un fluide visquex par un autre. Th?se de doctorat. Université de Toulouse, La Faculté des Science, France.

Barenblatt, G.I., and A.A. Gil'man. 1987. Nonequilibrium counterflow capillary impregnation. J. Eng. Phys. 52:335-339.

Bear, J., and A. Verruijt. 1987. Modeling groundwater flow and pollution. D. Reidel Publ. Co., Dordrecht, The Netherlands.

Beliaev, A.Y., and S.M. Hassanizadeh. 2001. A theoretical model of hysteresis and dynamic effects in the capillary relation for twophase flow in porous media. Transp. Porous Media 43:487-510.

Beliaev, A.Y., and R.J. Schotting. 2002. Analysis of a new model of unsaturated flow in porous media. Comput. Geosci. 5:345-368.

Biggar, J.W., and S.A. Taylor. 1960. Some aspects of the kinetics of moisture flow into unsaturated soil. Soil Sci. Soc. Am. Proc. 24: $81-85$.

Blom, S.M.P., and J. Hagoort. 1997. Relative permeability at nearcritical conditions. SPE Paper \# 38935. SPE Conference. San Antonio, TX. 5-8 Oct. 1997. SPE, Brookfield, CT.

Celia, M.A., E.T. Bouloutas, and R.L. Zarba. 1990. A general massconservative numerical solution for the unsaturated flow equation. Water Resour. Res. 26:1483-1496.

Celia, M.A., P.C. Reeves, and L.A. Ferrand. 1995. Recent advances in pore-scale models for multiphase flow in porous media. Rev. Geophys. Suppl. 1049-1057, July.

Constantinides, G.N., and A.C. Payatakes. 1996. Network simulation of steady-state two-phase flow in consolidated porous media. AIChE J. 42:369-382.

Crank, J. The mathematics of diffusion. 1956. Oxford Press, Oxford, UK.

Dahle, H.K., and M. Celia. 1999. A dynamic network model for twophase immiscible flow. Comput. Geosci. 3:1-22.

Davidson, J.M., D.R. Nielsen, and J.W. Biggar. 1966. The dependence of soil water uptake and release upon the applied pressure increment. Soil. Sci. Soc. Am. Proc. 30:298-304.

Dullien, A.F.L. 1992. Porous media: Fluid transport and pore structure. 2nd ed. Academic Press, New York, NY.

Durner, W., B. Schultze, and T. Zurmhl. 1996. Transient flow experiments for the determination of soil hydraulic properties-An evaluation of different boundary conditions. p. 85-88. In W. Durner et al. (ed.) European Workshop on Advanced Methods to Determine Hydraulic Properties of Soils. 10-12 June 1996. University of Bayreuth, Bayreuth, Germany.

Dussan, E.B.V. 1987. Incorporation of the influence of wettability into models of immiscible fluid displacements through a porous media. In Physics and Chemistry of Porous Media II, AIP Conference Proceedings 154. Amer. Inst. of Physics, College Park, MD.

Elrick, D.E. 1963. Unsaturated flow properties of soils. Austr. J. Soil Res. 1:1-8.

Elzeftawy, A., and R.S. Mansell. 1975. Hydraulic conductivity calculations for unsaturated steady-state and transeitn-state flow in sands. Soil Sci. Soc. Am. Proc. 39:599-603.

Friedman, S.P. 1999. Dynamic contact angle explanation of flow ratedependent saturation-pressure relationships during transient liquid flow in unsaturated porous media. J. Adhesion Sci. Technol. 13: $1495-1518$.

Gray, W.G., and S.M. Hassanizadeh. 1991a. Unsaturated flow theory including interfacial phenomena. Water Resour. Res. 27:1855-1863.

Gray, W.G., and S.M. Hassanizadeh. 1991b. Paradoxes and realities in unsaturated flow theory. Water Resour. Res. 27:1847-1854.

Hassanizadeh, S.M., and W.G. Gray. 1990. Mechanics and thermodynamics of multiphase flow in porous media including interphase boundaries. Adv. Water Resour. 13:169-186.

Hassanizadeh, S.M., and W.G. Gray. 1993a. Thermodynamic basis of capillary pressure in porous media. Water Resour. Res. 29:3389-3405.

Hassanizadeh, S.M., and W.G. Gray. 1993b. Toward an improved description of the physics of two-phase flow. Adv. Water Resour. 16:53-67.

Hillel, D. 1980. Fundamentals of soil physics. Academic Press, San Diego, CA.

Hollenbeck, K.J., and K.H. Jensen. 1998. Experimental evidence of randomness and non-uniqueness in unsaturated outflow experiments designed for hydraulic parameter estimation. Water Resour. Res. 34:595-602.

Kalaydjian, F. 1992a. A macroscopic description of multiphase flow in porous media involving space-time evolution of fluid-fluid interfaces. Transp. Porous Media. 2:491-506.

Kalaydjian, F. 1992b. Dynamic capillary pressure curve for water/oil displacement in porous media, theory vs. experiment. p. 491-506. Paper \#24813. SPE Conference. Washington, DC. 4-7 Oct. 1992 SPE, Brookfield, CT.

Kirkham, D., and L. Feng. 1949. Some testes of the diffusion theory, and laws of capillary flow, in soils. Soil Sci. 67:29-40.

Kool, J.B., J.C. Parker, and M.Th. van Genuchten. 1985. Determination of soil hydraulic properties from one-step outflow experiments by parameter estimation: I. Theory and numerical studies. Soil Sci. Soc. Am. J. 49:1348-1354.

Lake, L. 1989. Enhanced oil recovery. Prentice Hall, Englewood Cliffs, NJ.

Mokady, R.S., and P.F. Low. 1964. The tension-moisture content relationship under static and dynamic conditions. Soil Sci. Soc. Am. Proc. 28:583-584.

Nielsen, D.R., J.W. Biggar, and J.M. Davidson. 1962. Experimental consideration of diffusion analysis in unsaturated flow problems. Soil Sci. Soc. Am. Proc. 26:107-11.

Nützmann, G., H. Moser, and H. Handke. 1994. Inverse parameter identification of soil hydraulic properties results of a new soil column experiment. p. 785-792. In A. Peters et al. (ed.) Proceedings of Computational Methods in Water Resources X. Heidelberg, Germany. June 1994. Kluwer, Dordrecht, The Netherlands.

Plagge, R., P. Häupl, and M. Renger. 1999. Transient effects on the hydraulic properties of porous media. p. 905-912. In M.Th. van Genuchten et al. (ed.) Proc. Int'l. Workshop, Characterization and Measurement of the Hydraulic Properties of Unsaturated Porous Media. University of California, Riverside, CA.

Ramakrishnan, T.S., and D.T. Wasan. 1986. Effect of capillary number on the relative permeability function for two-phase flow in porous media. Powder Technol. 48:99-124.

Rawlins, S.L., and W.H. Gardner. 1963. A test of the validity of the diffusion equation for unsaturated flow of soil water. Soil Sci. Soc. Am. Proc. 27:507-511.

Schultze, B., O. Ippisch, B. Huwe, and W. Durner. 1999. Dynamic nonequilibrium during unsaturated water flow. p. 877-892. In M.Th. van Genuchten et al. (ed.) Proc. Int'l. Workshop, Characterization and Measurement of the Hydraulic Properties of Unsaturated Porous Media. University of California, Riverside, CA.

Smiles, D.E., G. Vachaud, and M. Vauclin. 1971. A test of the uniqueness of the soil moisture characteristic during transient, non-hysteretic flow of water in a rigid soil. Soil Sci. Soc. Am. Proc. 35:535-539.

Stauffer, F. 1978. Time dependence of the relations between capillary pressure, water content and conductivity during drainage of porous media. IAHR Symp. on Scale Effects in Porous Media. Thessaloniki, Greece. 29 Aug.-1 Sept. 1978. IAHR, Madrid, Spain.

Stephens, D.B. 1995. Vadose zone hydrology. Lewis Publ., Boca Raton, FL.

Topp, G.C., A. Klute, and D.B. Peters. 1967. Comparison of water content-pressure head data obtained by equilibrium, steady-state, and unsteady-state methods. Soil Sci. Soc. Am. Proc. 31:312-314.

Vachaud, G., M. Vauclin, and M. Wakil. 1972. A study of the uniqueness of the soil moisture characteristic during desorption by vertical drainage. Soil Sci. Soc. Am. Proc. 36:531-532.

Watson, K.K. 1965. Non-continuous porous media flow. Report No. 84. Water Research Laboratory, The University of New South Wales, Manly Vale, Australia.

Watson, K.K., and F.D. Whisler. 1968. System dependence of the water content-pressure head relationship. Soil. Sci. Soc. Am. Proc. 32:121-123.

Watson, K.K., and F.D. Whisler. 1978. The use of dynamic soil water characteristics in a numerical desorption model. Soil. Sci. 125:83-91.

Wanna-Etyem, C. 1982. Static and dynamic water content-pressure head relations of porous media. Ph.D. diss. Colorado State University, Fort Collins, CO.

Wildenschild, D., and J.W. Hopmans. 1999. Flow rate dependence of hydraulic properties of unsaturated porous media. p. 893-904. In M.Th. van Genuchten et al. (ed.) Proc. Int'l. Workshop, Characterization and Measurement of the Hydraulic Properties of Unsaturated Porous Media. University of California, Riverside, CA.

Wildenschild, D., J.W. Hopmans, and J. Simunek. 2001. Flow rate dependence of soil hydraulic characteristics. Soil Sci. Soc. Am. J. 65:35-48 